\title{
Attractors for a Class of Abstract Evolution Equations with Fading Memory
}

\author{
Xuan Wang, Fenxia Duan, and Didi Hu \\ College of Mathematics and Statistics, Northwest Normal University, Lanzhou 730070, China \\ Correspondence should be addressed to Xuan Wang; wangxuan@nwnu.edu.cn \\ Received 1 January 2017; Accepted 27 April 2017; Published 14 June 2017 \\ Academic Editor: Kishin Sadarangani
}

Copyright (C) 2017 Xuan Wang et al. This is an open access article distributed under the Creative Commons Attribution License, which permits unrestricted use, distribution, and reproduction in any medium, provided the original work is properly cited.

In this paper, we study the dynamics of an abstract evolution equation with fading memory with a critical growing nonlinearity. By use of some new methods and asymptotic estimate techniques, we first verify the asymptotic compact of solution semigroup and then prove the existence of global attractors in weak topological space and strong topological space, while the forcing term only belongs to $H^{-1}(\Omega)$ or $L^{2}(\Omega)$, respectively. The results are new and appear to be optimal.

\section{Introduction}

In this paper, we consider the asymptotic behaviors of solutions for the following abstract evolution equation with fading memory in a bounded domain $\Omega \subset \mathbb{R}^{n}$ :

$$
\begin{aligned}
& u_{t t}+\alpha u_{t}+k(0) A^{\theta} u+\int_{0}^{\infty} k^{\prime}(s) A^{\theta} u(t-s) d s+f(u) \\
& \quad=g(x), \quad \Omega \times \mathbb{R}^{+}, \\
& u(x, t)=0, \quad x \in \partial \Omega, t \in \mathbb{R}, \\
& u(x, t)=u_{0}(x, t), \quad x \in \Omega, t \leqslant 0,
\end{aligned}
$$

where $\theta \in[2 n /(n+2), n / 2)$.

About the forcing term $g$, we consider two cases: the weak solutions case $g(x) \in H^{-1}(\Omega)$ and the strong solutions case $g(x) \in L^{2}(\Omega)$. For the nonlinearity, we assume that $f \in$ $C^{1}(\mathbb{R})$ with $f(0)=0$ and satisfies the following conditions.

Dissipation condition:

$$
\limsup _{|s| \rightarrow \infty} \frac{f(s)}{s}>-\lambda_{1}^{\theta}
$$

where $\lambda_{1}$ is the first eigenvalue of $A=-\Delta$ in $H_{0}^{1}(\Omega)$.
Growth condition:

$$
\begin{aligned}
& \left|f^{\prime}(s)\right| \leqslant C\left(1+|s|^{p}\right), \\
& \forall s \in \mathbb{R}, \begin{cases}p \leqslant \frac{4}{n-2}, & n \geqslant 3, \\
p \geqslant 0 \text { is arbitrary, } & n=1,2 .\end{cases}
\end{aligned}
$$

Since the nonlinearities critical exponent is $4 /(n-4)$ in the strong topological space, we need $f$ to satisfy the following condition instead of (3):

$$
\begin{aligned}
& \left|f^{\prime}(s)\right| \leqslant C\left(1+|s|^{p}\right), \\
& \forall s \in \mathbb{R}, \begin{cases}p \leqslant \frac{4}{n-4}, & n \geqslant 5, \\
p \geqslant 0 \text { is arbitrary, } & n=3,4,\end{cases}
\end{aligned}
$$

$f(s) s \geqslant 0, \quad \forall s \in \mathbb{R}$.

And there exists a constant $l>0$, such that

$$
f^{\prime}(s) \leqslant l, \quad \forall s \in \mathbb{R},
$$

and assumption (6) about $f$ will be used to prove the existence of absorbing set in the strong topological space and that it has nothing to do with the testing of asymptotic compactness. 
There are many functions satisfying the above assumption (6). For example, when $f(u)=u-u^{3}, f(u)$ satisfies this condition. Without loss of generality, we also assume $f(0)=0$.

The effects of fading memory in this equation are shown through the linear time convolution of the function $A^{\theta} u(\cdot)$ and memory kernel $k(\cdot)$. As that in [1], we also assume that the memory kernel $k(\cdot) \in C^{2}\left(\mathbb{R}^{+}\right), k(s) \geqslant 0, k^{\prime}(s) \leqslant 0$, for every $s \in \mathbb{R}^{+}$. Besides, we also presume that the function $\mu(s)=$ $-k^{\prime}(s)$ satisfies the following hypotheses:

$$
\begin{gathered}
\mu \in C^{1}\left(\mathbb{R}^{+}\right) \cap L^{1}\left(\mathbb{R}^{+}\right), \\
\mu(s) \geqslant 0, \mu^{\prime}(s) \leqslant 0, \forall s \in \mathbb{R}^{+} ; \\
\int_{0}^{\infty} \mu(s) d s=k_{0}>0 ; \\
\mu^{\prime}(s)+\delta \mu(s) \leqslant 0, \quad \forall s \geqslant 0,
\end{gathered}
$$

where $\delta$ is a positive constant. Obviously, it follows that the kernel $\mu(s)$ decays to zero with exponential rate. This behavior displays the fading memory of the far history in model (1).

If $\theta=1$ and $A=-\Delta$, (1) represents a semilinear hyperbolic equation in viscoelasticity (cf. $[2,3])$, and this model is originally derived from the theory of isothermal viscoelasticity and describes a process of energy dissipation of a homogeneous and isotropic viscoelastic solid (cf. [4]). Furthermore, if $k^{\prime}(s) \equiv 0$, (1) reduces to semilinear wave equation, where $g$ denotes some displacement-dependant body's force density (cf. [5]); and, under these conditions, if $f(u)=\sin u$, (1) can be transformed into a model of Sine-Gordon equation (cf. [6]); if $f(u)=|u|^{\gamma} u$, (1) can be turned into the relative quantum mechanical equation (cf. [6]). If $k^{\prime}(s) \equiv 0, \theta=2$, and $A=-\Delta$, it expresses a floating beam equation; $u$ denotes bent movement crosswise of beam (cf. $[7,8])$.

For the semilinear hyperbolic equation in viscoelasticity, the asymptotic behaviors of the solution have been studied in $[1,9-13]$. In $[1,9,10]$, the authors investigated the asymptotic behaviors of solutions when nonlinearity is subcritical growth. In [11], Sun et al. considered large-time behaviors of the solutions and gained the existence of global attractors for nonautonomous strongly damped wave-type evolution equation with the critical nonlinearity and linear memory. In [12], Cavalcanti et al. studied the long-time dynamics of a semilinear wave equation with degenerate viscoelasticity defined in a bounded domain $\Omega$ of $\mathbb{R}^{3}$, with Dirichlet boundary condition and nonlinear forcing term with critical growth. In [13], Zhou and Zhao proved the existence of random attractors for the continuous random dynamical systems generated by stochastic damped nonautonomous wave equations with linear memory and additive white noise when the nonlinearity has a critically growing exponent and studied the upper semicontinuity of random attractors.

For the floating beam equation, the authors considered the dynamics and obtained the existence of a global attractor for the deterministic floating beam in [8] and deeply studied the existence of a compact random attractor for the random dynamical system generated by a model for nonlinear oscillations in a floating beam equation with strong damping and white noise in [14].
The above equations are all special cases of an abstract evolution equation with fading memory that we will study in this paper. Until now, we find that no one else has studied the long-time behavior of the solutions about the problem with weak damping and critical nonlinearity, which just causes our strong research motivation and interest. Based on this, we will study the asymptotic behaviors and regularity of solutions for this problem in this paper.

As we know, if we want to prove the existence of global attractors, the key point is to obtain the compactness of the semigroup in some sense. However, there exist some essential difficulties to test the continuity and compactness (asymptotic compactness) of the semigroup of solutions for (1). First, due to the memory terms, we cannot use $\left(I-P_{m}\right) u$ or $(I-$ $\left.P_{m}\right) A^{\theta} u$ as test function to verify the asymptotic compactness of the solution semigroup; second, the critical nonlinearity leads to the fact that some obstacles are hard to avoid in the process of energy estimation. Furthermore, since $A^{\theta}$ is an abstract operator, it brings about more substantive barriers in the process of proof. Despite all this, we still overcome the above bottlenecks in the process of estimation and proof by applying semigroup theory and decomposition technology. Finally, we test the continuity and compactness (asymptotic compactness) of the solution semigroup of solutions and obtain the existence of global attractors of (1) in the weak topological space $V_{\theta} \times L^{2}(\Omega) \times L_{\mu}^{2}\left(\mathbb{R}^{+}, V_{\theta}\right)$ and the strong topological space $V_{2 \theta} \times V_{\theta} \times L_{\mu}^{2}\left(\mathbb{R}^{+}, V_{2 \theta}\right)$.

This paper is organized as follows. Some preliminaries, including some notations that we will use, the assumption on nonlinearity, and some general abstract results about dynamical system are presented in Section 2. Then, the proof of our main result about compactness testing and the existence of global attractor for the dynamical system generated by the solution of (1) are given in Sections 3 and 4. The main results are Theorems 11 and 18.

\section{Preliminaries}

Let $H=L^{2}(\Omega)$ and $b(u, v)$ be a bilinear continuous form on $H$ which is symmetric and coercive. With this form, we associate the linear operator $A$ in $H$ by setting $(A u, v)=$ $b(u, v), \forall u, v \in H$, and $A$ can be considered as a self-adjoint unbounded operator in $H$ with domain $D(A) \subset H$.

We assume that $\left\{\lambda_{j}\right\}_{j \in N},\left\{\omega_{j}\right\}_{j \in N}$ are eigenvalue and eigenvectors of $A$, so $\left\{\omega_{j}\right\}_{j \in N}$ can form a group of orthonormal bases of $H$; then

$$
\begin{aligned}
A \omega_{j} & =\lambda_{j} \omega_{j}, \quad \forall j \in \mathbb{N}, \\
0 & <\lambda_{1} \leqslant \lambda_{2} \leqslant \cdots \leqslant \lambda_{j}, \quad \lambda_{j} \longrightarrow \infty, \text { as } j \longrightarrow \infty .
\end{aligned}
$$

Using this group of bases we can easily define the powers $A^{\theta}$ of $A$ (see [6]), $\theta \in[2 n /(n+2), n / 2)$, with domain $D\left(A^{\theta}\right) \subset$ $H$. we can consider a family of Hilbert spaces

$$
\begin{aligned}
D\left(A^{\theta}\right)=\left\{u \in H, \sum_{j=1}^{\infty} \lambda_{j}^{2 \theta}\left(u, \omega_{j}\right)^{2}<\infty\right\}, & \\
& \theta \in\left[\frac{2 n}{n+2}, \frac{n}{2}\right),
\end{aligned}
$$


with the standard inner product and norms, respectively,

$$
\begin{gathered}
\langle\cdot, \cdot\rangle_{D\left(A^{\theta}\right)}=\left\langle A^{\theta} \cdot, A^{\theta} \cdot\right\rangle, \\
\|\cdot\|_{D\left(A^{\theta}\right)}^{2}=\left\|A^{\theta} \cdot\right\|^{2} ;
\end{gathered}
$$

here $\langle\cdot, \cdot\rangle$ and $\|\cdot\|$ mean $L^{2}(\Omega)$ inner product and norm, respectively. Then, we know that

$$
\begin{gathered}
D\left(A^{\theta_{1}}\right) \hookrightarrow D\left(A^{\theta_{2}}\right), \quad \text { for any } \theta_{1}>\theta_{2}, \\
D\left(A^{\theta / 2}\right) \hookrightarrow L^{2 n /(n-2 \theta)}(\Omega), \quad \forall \theta \in\left[0, \frac{n}{2}\right) .
\end{gathered}
$$

The following interpolation inequality also holds: given $s>$ $r>q$, for any $\epsilon>0$, there exists a positive constant $C_{\epsilon}=$ $C_{\epsilon}(s, r, q)$ such that

$$
\begin{aligned}
& \left\|A^{r / 2} u\right\| \leqslant \epsilon\left\|A^{s / 2} u\right\|+C_{\epsilon}\left\|A^{q / 2} u\right\|, \\
& \text { for any } u \in D\left(A^{s / 2}\right) .
\end{aligned}
$$

For convenience, we set, for $s \in[2 n /(n+2), n / 2)$,

$$
V_{s}=D\left(A^{s / 2}\right) \text {, with norm }\|\cdot\|_{V_{s}}=\|\cdot\|_{D\left(A^{s / 2}\right)} \text {; }
$$

then, $V_{0}=H=L^{2}(\Omega), V_{\theta}=D\left(A^{\theta / 2}\right)$, and $V_{2 \theta}=D\left(A^{\theta}\right)$.

As in $[1,9]$, we denote

$$
\eta^{t}(x, s)=u(x, t)-u(x, t-s) .
$$

Set $\mu(s)=-k^{\prime}(s)$ and use the assumption $k(\infty)=1 ;(1)$ can be transformed into the following system:

$$
\begin{aligned}
u_{t t} & +\alpha u_{t}+A^{\theta} u+\int_{0}^{\infty} \mu(s) A^{\theta} \eta^{t}(s) d s+f(u) \\
& =g(x), \\
\eta_{t}^{t} & =-\eta_{s}^{t}+u_{t} .
\end{aligned}
$$

The associated initial-boundary conditions are

$$
\begin{aligned}
u(x, t) & =0, \\
\eta^{t}(x, s) & =0, \\
u(x, t) & =u_{0}(x, t), \quad(x, s) \in \partial \Omega \times \mathbb{R}^{+}, t \geqslant 0, \\
\partial_{t} u(x, t) & =\partial_{t} u_{0}(x, t), \quad(x, t) \in \Omega \times(-\infty, 0], \\
\eta^{0}(x, s) & =u_{0}(x, 0)-u_{0}(x,-s), \quad(x, s) \in \Omega \times \mathbb{R}^{+},
\end{aligned}
$$

where $u(\cdot)$ satisfies the condition as follows: there exist two positive constants $\mathscr{R}$ and $\varrho=\min \left\{\delta / 2, \lambda_{1} / 2\right\}$; here $\lambda_{1}$ is the first eigenvalue of $-\Delta$, such that

$$
\int_{0}^{\infty} e^{-\varrho s}\|\nabla u(-s)\|^{2} d s \leqslant \mathscr{R}
$$

here $\|\cdot\|$ means $L^{2}(\Omega)$ norm.

In view of (7), we let $L_{\mu}^{2}\left(\mathbb{R}^{+} ; V_{\theta}\right)$ be a family of Hilbert spaces of $V_{\theta}$-valued functions on $\mathbb{R}^{+}$and $L_{\mu}^{2}\left(\mathbb{R}^{+} ; V_{2 \theta}\right)$ be a family of Hilbert spaces of $V_{2 \theta^{-}}$valued functions on $\mathbb{R}^{+}$, endowed with the following inner product and norm, respectively:

$$
\begin{aligned}
\langle\varphi, \psi\rangle_{\mu, V_{\theta}} & =\int_{0}^{\infty} \mu(s) \int_{\Omega} A^{\theta / 2} \varphi A^{\theta / 2} \psi d x d s, \\
\|\varphi\|_{\mu, V_{\theta}}^{2} & =\int_{0}^{\infty} \mu(s) \int_{\Omega}\left|A^{\theta / 2} \varphi\right|^{2} d x d s, \\
\langle\varphi, \psi\rangle_{\mu, V_{2 \theta}} & =\int_{0}^{\infty} \mu(s) \int_{\Omega} A^{\theta} \varphi A^{\theta} \psi d x d s, \\
\|\varphi\|_{\mu, V_{2 \theta}}^{2} & =\int_{0}^{\infty} \mu(s) \int_{\Omega}\left|A^{\theta} \varphi\right|^{2} d x d s .
\end{aligned}
$$

So, we introduce the family of Hilbert spaces:

$$
\begin{aligned}
& \mathscr{H}_{1}=V_{\theta} \times H \times L_{\mu}^{2}\left(\mathbb{R}^{+} ; V_{\theta}\right) ; \\
& \mathscr{H}_{2}=V_{2 \theta} \times V_{\theta} \times L_{\mu}^{2}\left(\mathbb{R}^{+} ; V_{2 \theta}\right)
\end{aligned}
$$

and endow this family of spaces with norm, respectively,

$$
\begin{aligned}
\|z\|_{\mathscr{H}_{1}} & =\left\|\left(u, u_{t}, \eta^{t}\right)\right\|_{\mathscr{H}_{1}} \\
& =\left(\frac{1}{2}\left(\|u\|_{V_{\theta}}^{2}+\left\|u_{t}\right\|^{2}+\left\|\eta^{t}\right\|_{\mu, V_{\theta}}^{2}\right)\right)^{1 / 2} ; \\
\|z\|_{\mathscr{H}_{2}} & =\left\|\left(u, u_{t}, \eta^{t}\right)\right\|_{\mathscr{H}_{2}} \\
& =\left(\frac{1}{2}\left(\|u\|_{V_{2 \theta}}^{2}+\left\|u_{t}\right\|_{V_{\theta}}^{2}+\left\|\eta^{t}\right\|_{\mu, V_{2 \theta}}^{2}\right)\right)^{1 / 2} .
\end{aligned}
$$

In order to estimate conveniently, using assumptions (7)-(9), we can obtain the preliminary result as follows (cf. $[9,15])$.

Lemma 1. Set $I=[0, T], \forall T>0$. If memory kernel $\mu(s)$ satisfies (7)-(9), then, for any $\eta^{t} \in C^{1}\left(I ; L_{\mu}^{2}\left(\mathbb{R}^{+} ; V_{r}\right)\right), 0<r<$ $2 \theta, \theta \in[2 n /(n+2), n / 2)$, there exists a constant $\delta>0$, such that

$$
\left\langle\eta^{t}, \eta_{s}^{t}\right\rangle_{\mu, V_{r}} \geqslant \frac{\delta}{2}\left\|\eta^{t}\right\|_{\mu, V_{r}}^{2} .
$$

The following results will be used to prove the compactness of the memory term and the existence of strong global attractors.

Lemma 2 (see $[9,15,16])$. Assume that $\mu \in C^{1}\left(\mathbb{R}^{+}\right) \cap L^{1}\left(\mathbb{R}^{+}\right)$ is a nonnegative function and satisfies the following: if there exists $s_{0} \in \mathbb{R}^{+}$, such that $\mu\left(s_{0}\right)=0$, then $\mu(s)=0$ for all $s \geqslant s_{0}$ holds. Moreover, let $B_{0}, B_{1}, B_{2}$ be Banach space, where $B_{0}, B_{1}$ are reflexive and satisfy

$$
B_{0} \hookrightarrow B_{1} \hookrightarrow B_{2},
$$

where the embedding $B_{0} \hookrightarrow B_{1}$ is compact. Let $\mathscr{C} \subset L_{\mu}^{2}\left(\mathbb{R}^{+}\right.$; $\left.B_{1}\right)$ and satisfy

(i) $\mathscr{C}$ in $L_{\mu}^{2}\left(\mathbb{R}^{+} ; B_{0}\right) \cap H_{\mu}^{1}\left(\mathbb{R}^{+} ; B_{2}\right)$;

(ii) $\sup _{\eta \in \mathscr{C}}\|\eta(s)\|_{B_{1}}^{2} \leqslant h(s), \forall s \in \mathbb{R}^{+}, h(s) \in L_{\mu}^{1}\left(\mathbb{R}^{+}\right)$.

Then $\mathscr{C}$ is relatively compact in $L_{\mu}^{2}\left(\mathbb{R}^{+} ; B_{1}\right)$. 
Lemma 3 (see [6]). Let H be a complete metric space, $\{S(t)\}_{t \geqslant 0}$ is a $C^{0}$ semigroup in $H$, and $\{S(t)\}_{t \geqslant 0}$ has a bounded absorbing set $B_{0}$ in $H$. If, for every $t \geqslant 0$, the operator $S(t)$ allows the decomposition $S(t)=S_{1}(t)+S_{2}(t)$ and satisfies that

(i) the semigroup $\left\{S_{1}(t)\right\}_{t \geqslant 0}$ is uniformly compact, as $t$ is increasing sufficiently;

(ii) the operator $S_{2}(t): H \rightarrow H$ is continuous and, for any bounded set $B \subset H$, as $t \rightarrow \infty$,

$$
r_{B}(t)=\sup _{\varphi \in B}\left\|S_{2}(t) \varphi\right\|_{H} \longrightarrow 0,
$$

then $\omega$-limit set of absorbing set $B_{0}$ is global attractors of $\{S(t)\}_{t \geqslant 0}$.

\section{Global Attractors in $\mathscr{H}_{1}$}

3.1. The Existence and Uniqueness of Weak Solution. At first, we define the concept of weak solution for dynamical systems (17)-(18) (cf. [15] about the corresponding definition of solutions for wave equations).

Definition 4. Define $I=[0, T], \forall T>0$. Let $g \in H^{-1}(\Omega)$ and $z_{0} \in \mathscr{H}_{2}$. A ternary form $z=\left(u, u_{t}, \eta^{t}\right)$ which fulfils

$$
\begin{aligned}
u & \in C\left(I ; V_{\theta}\right), \\
u_{t} & \in L^{2}\left(I ; V_{\theta}\right), \\
\eta^{t} & \in C\left(I ; L_{\mu}^{2}\left(\mathbb{R}^{+} ; V_{\theta}\right)\right) ; \\
\eta_{t}^{t}+\eta_{s}^{t} & \in L^{\infty}\left(I ; L_{\mu}^{2}\left(\mathbb{R}^{+} ; H\right)\right) \cap L^{2}\left(I ; L_{\mu}^{2}\left(\mathbb{R}^{+} ; V_{\theta}\right)\right)
\end{aligned}
$$

is said to be a weak solution of problem (17)-(18) in the time interval $I$ with initial data $z(0)=z_{0}$, provided that

$$
\begin{aligned}
& \left\langle u_{t t}, \omega\right\rangle+\alpha\left\langle u_{t}, \omega\right\rangle+\langle u, \omega\rangle_{V_{\theta}}+\left\langle\eta^{t}, \omega\right\rangle_{\mu, V_{\theta}} \\
& +\langle f(u), \omega\rangle=\langle g, \omega\rangle, \\
& \left\langle\eta_{t}^{t}+\eta_{s}^{t}, \varphi\right\rangle_{\mu, V_{\theta}}=\left\langle u_{t}, \varphi\right\rangle_{\mu, V_{\theta}},
\end{aligned}
$$

for all $\omega \in V_{\theta}, \varphi \in L_{\mu}^{2}\left(\mathbb{R}^{+} ; V_{\theta}\right)$, a.e. $t \in I$.

Then, we start with the following general existence and unique result. The proof is based on the normal FaedoGalerkin methods introduced in $[9,15]$.

Theorem 5 (existence and uniqueness of solutions). Let $f$ satisfy (2)-(3), $g \in H^{-1}(\Omega)$, and (7)-(9) hold. Then for any given $z_{0}=\left(u_{0}, u_{t 0}, \eta^{0}\right) \in \mathscr{H}_{1}$, and any $T>0$, there is a unique solution $z(t)=\left(u, u_{t}, \eta^{t}\right) \in \mathscr{H}_{1}$ for problem (17)-(18), satisfying

$$
z \in L^{\infty}\left([0, T] ; \mathscr{H}_{1}\right) \cap C\left([0, T] ; \mathscr{H}_{1}\right) .
$$

According to Theorem 5 above, we can define the solution operator; that is,

$$
\begin{aligned}
S(t): \mathscr{H}_{1} & \longrightarrow \mathscr{H}_{1}, \\
z_{0} & =\left(u_{0}, u_{t 0}, \eta^{0}\right) \longrightarrow \\
\left(u(t), u_{t}(t), \eta^{t}\right) & =S(t) z_{0} .
\end{aligned}
$$

Obviously, $\{S(t)\}_{t \geqslant 0}$ can form a solution semigroup.

In the remainder of this section, we denote by $\{S(t)\}_{t \geqslant 0}$ the semigroup associated with the solution of (17)-(18).

\subsection{The Existence of Bounded Absorbing Set}

Theorem 6 (bounded absorbing in $\mathscr{H}_{1}$ ). Let $B_{0}$ be any bounded subset of $\mathscr{H}_{1}$ and $z(t)$ be a solution of (17)-(18) with initial data $z_{0} \in B_{0}$. If the nonlinearity $f$ satisfies (2), $g \in$ $H^{-1}(\Omega)$, (7)-(9) hold. Then there is a positive constant $\mu_{0}$ such that, for any bounded subset $B_{0} \subset \mathscr{H}_{1}$, there exists $t_{0}=$ $t_{0}\left(\left\|B_{0}\right\|_{\mathscr{C}_{1}}\right)$ such that

$$
\left\|S(t) z_{0}\right\|_{\mathscr{H}_{1}} \leqslant \mu_{0}, \quad \forall t \geqslant t_{0}, z_{0} \in B_{0} .
$$

Proof. Taking $0<\rho<1$, multiplying (17) by $u_{t}+\rho u$, and integrating over $\Omega$, we have

$$
\begin{aligned}
\frac{1}{2} \frac{d}{d t}( & \left.\|u\|_{V_{\theta}}^{2}+\left\|u_{t}+\rho u\right\|^{2}+\left\|\eta^{t}\right\|_{\mu, V_{\theta}}^{2}\right) \\
& +(\alpha-\rho)\left\|u_{t}+\rho u\right\|^{2}+\rho\|u\|_{V_{\theta}}^{2}+\left\langle\eta^{t}, \eta_{s}^{t}\right\rangle_{\mu, V_{\theta}} \\
& -\rho(\alpha-\rho)\left\langle u, u_{t}+\rho u\right\rangle+\rho\left\langle\eta^{t}, u\right\rangle_{\mu, V_{\theta}} \\
& +\left\langle f(u), u_{t}+\rho u\right\rangle=\left\langle g, u_{t}+\rho u\right\rangle .
\end{aligned}
$$

Then, using Poincaré inequality and Cauchy inequality, and taking $\rho$ small enough, we can get that

$$
\begin{gathered}
\rho\|u\|_{V_{\theta}}^{2}+(\alpha-\rho)\left\|u_{t}+\rho u\right\|^{2}-\rho(\alpha-\rho)\left\langle u, u_{t}+\rho u\right\rangle \\
\geqslant \rho\|u\|_{V_{\theta}}^{2}+(\alpha-\rho)\left\|u_{t}+\rho u\right\|^{2}-\rho \alpha\left\langle u, u_{t}+\rho u\right\rangle \\
+\rho^{2}\left\langle u, u_{t}\right\rangle \\
\geqslant \rho\|u\|_{V_{\theta}}^{2}+(\alpha-\rho)\left\|u_{t}+\rho u\right\|^{2}-\frac{\alpha \rho^{2}}{\lambda_{1}^{\theta}}\|u\|_{V_{\theta}}^{2} \\
\quad-(\alpha-\rho)\left\langle\rho u, u_{t}\right\rangle \\
\geqslant \rho\|u\|_{V_{\theta}}^{2}+(\alpha-\rho)\left\|u_{t}+\rho u\right\|^{2}-\frac{\alpha \rho^{2}}{\lambda_{1}^{\theta}}\|u\|_{V_{\theta}}^{2} \\
\quad-\frac{\alpha}{2}\left\|u_{t}+\rho u\right\|^{2} \\
=\rho\left(1-\frac{\alpha \rho}{\lambda_{1}^{\theta}}\right)\|u\|_{V_{\theta}}^{2}+\left(\frac{1}{2} \alpha-\rho\right)\left\|u_{t}+\rho u\right\|^{2} .
\end{gathered}
$$

Applying Lemma 1, we have

$$
\left\langle\eta^{t}, \eta_{s}^{t}\right\rangle_{\mu, V_{\theta}} \geqslant \frac{\delta}{2}\left\|\eta^{t}\right\|_{\mu, V_{\theta}}^{2},
$$


and, from (8), we see

$$
\begin{aligned}
& \rho\left\langle\eta^{t}, u\right\rangle_{\mu, V_{\theta}} \geqslant-\rho \int_{\Omega}\left(\int_{0}^{\infty} \mu(s)\left|A^{\theta / 2} \eta^{t}(s)\right|^{2} d s\right)^{1 / 2} \\
& \cdot\left(\int_{0}^{\infty} \mu(s)\left|A^{\theta / 2} u\right|^{2} d s\right)^{1 / 2} d x \geqslant-\frac{\delta}{4}\left\|\eta^{t}\right\|_{\mu, V_{\theta}}^{2} \\
& -\frac{k_{0} \rho^{2}}{\delta}\|u\|_{V_{\theta}}^{2} .
\end{aligned}
$$

Therefore,

$$
\begin{aligned}
\frac{1}{2} \frac{d}{d t}( & \left.\|u\|_{V_{\theta}}^{2}+\left\|u_{t}+\rho u\right\|^{2}+\left\|\eta^{t}\right\|_{\mu, V_{\theta}}^{2}\right) \\
& +\rho\left(1-\frac{\alpha \rho}{\lambda_{1}^{\theta}}-\frac{k_{0} \rho}{\delta}\right)\|u\|_{V_{\theta}}^{2} \\
& +\left(\frac{1}{2} \alpha-\rho\right)\left\|u_{t}+\rho u\right\|^{2}+\frac{\delta}{4}\left\|\eta^{t}\right\|_{\mu, V_{\theta}}^{2} \\
& +\left\langle f(u), u_{t}+\rho u\right\rangle \leqslant\left\langle g, u_{t}+\rho u\right\rangle .
\end{aligned}
$$

From (2), we know that there exist $\lambda_{1}^{\theta}>\lambda^{*}>0$ and $C_{0}>$ 0 such that

$$
\langle f(u), u\rangle \geqslant \frac{\lambda^{*}}{4} \int_{\Omega} F(u) d x-C_{0}
$$

here, $F(u)=\int_{0}^{u} f(r) d r$. Then

$$
\begin{aligned}
\left\langle f(u), u_{t}+\rho u\right\rangle \geqslant & \frac{d}{d t} \int_{\Omega} F(u) d x+\frac{\rho \lambda^{*}}{4} \int_{\Omega} F(u) d x \\
& -\rho C_{0} .
\end{aligned}
$$

Evidently, we can get

$$
\begin{aligned}
\left\langle g, u_{t}+\rho u\right\rangle & =\frac{d}{d t}\langle g, u\rangle+\rho\langle g, u\rangle \\
\langle g, u\rangle & \leqslant\|g\|_{H^{-1}(\Omega)}\|\nabla u\| \\
& \leqslant \frac{2}{\lambda_{1}^{\theta-1}}\|g\|_{H^{-1}(\Omega)}^{2}+\frac{\lambda_{1}^{\theta-1}}{8}\|\nabla u\|^{2} \\
& \leqslant \frac{2}{\lambda_{1}^{\theta-1}}\|g\|_{H^{-1}(\Omega)}^{2}+\frac{1}{8}\|u\|_{V_{\theta}}^{2} .
\end{aligned}
$$

Choose $\rho$ to be sufficiently small, such that

$$
\begin{array}{r}
1-\frac{\alpha \rho}{\lambda_{1}^{\theta}}-\frac{k_{0} \rho}{\delta} \geqslant \frac{1}{2}, \\
\frac{1}{2} \alpha-\rho \geqslant \frac{\alpha}{4} ;
\end{array}
$$

thus,

$$
\begin{aligned}
& \rho\left(1-\frac{\alpha \rho}{\lambda_{1}^{\theta}}-\frac{k_{0} \rho}{\delta}\right)\|u\|_{V_{\theta}}^{2}+\left(\frac{1}{2} \alpha-\rho\right)\left\|u_{t}+\rho u\right\|^{2} \\
& \geqslant \frac{\rho}{2}\|u\|_{V_{\theta}}^{2}+\frac{\alpha}{4}\left\|u_{t}+\rho u\right\|^{2} .
\end{aligned}
$$

Substituting (32)-(40) into (31), we can obtain that

$$
\begin{aligned}
& \frac{1}{2} \frac{d}{d t}\left(\|u\|_{V_{\theta}}^{2}+\left\|u_{t}+\rho u\right\|^{2}+\left\|\eta^{t}\right\|_{\mu, V_{\theta}}^{2}+2 \int_{\Omega} F(u) d x\right. \\
& \quad-2\langle g, u\rangle+\widetilde{C})+\frac{\beta_{0}}{2}\left(\|u\|_{V_{\theta}}^{2}+\left\|u_{t}+\rho u\right\|^{2}\right. \\
& \left.+\left\|\eta^{t}\right\|_{\mu, V_{\theta}}^{2}+2 \int_{\Omega} F(u) d x-2\langle g, u\rangle+\widetilde{C}\right) \leqslant \rho C_{0} \\
& \quad+\frac{\beta_{0}}{2} \widetilde{C} \leqslant C
\end{aligned}
$$

where $\beta_{0}=\min \left\{\rho, \alpha / 2, \delta / 2, \rho \lambda^{*} / 4\right\}$ and $\widetilde{C}=2 C_{0}+$ $\left(4 / \lambda_{1}^{\theta-1}\right)\|g\|_{H^{-1}(\Omega)}^{2}$.

Hence, from (36)-(38), there exist positive constants $C_{1}$, $C_{2}$, such that

$$
\begin{aligned}
& C_{1}\|z(t)\|_{\mathscr{H}_{1}}^{2} \leqslant \frac{1}{2}\left(\|u\|_{V_{\theta}}^{2}+\left\|u_{t}+\rho u\right\|^{2}+\left\|\eta^{t}\right\|_{\mu, V_{\theta}}^{2}\right. \\
& \left.\quad+2 \int_{\Omega} F(u) d x-2\langle g, u\rangle+\widetilde{C}\right) \leqslant C_{2}\|z(t)\|_{\mathscr{H}_{1}}^{2} .
\end{aligned}
$$

According to Gronwall lemma,

$$
\|z(t)\|_{\mathscr{H}_{1}}^{2} \leqslant \frac{C_{2}}{C_{1}}\|z(0)\|_{\mathscr{H}_{1}}^{2} e^{-\beta_{0} t}+\frac{C}{\beta_{0} C_{1}} .
$$

Assuming that $\|z(0)\|_{\mathscr{H}_{1}}^{2} \leqslant R$, as $t \geqslant t_{0}=t_{0}\left(\left\|B_{0}\right\|_{\mathscr{H}_{1}}\right)$, we have

$$
\|z(t)\|_{\mathscr{H}_{1}} \leqslant \mu_{0} .
$$

We complete the proof.

3.3. The Existence of Global Attractor in $\mathscr{H}_{1}$. Now we will establish the necessary asymptotic smoothness, similar to that in [17-19]; we also need to make some decompositions about nonlinearity, forcing term, solution, and solution semigroup.

About forcing term $g(x)$, we think about two cases: the weak solutions case $g(x) \in H^{-1}(\Omega)$ : for every $g \in H^{-1}(\Omega)$ and any $\epsilon>0$, there is $g^{\epsilon} \in L^{2}(\Omega)$ which depends on $g$ and $\epsilon$, such that

$$
\left\|g-g^{\epsilon}\right\|_{H^{-1}(\Omega)}<\epsilon
$$

the strong solutions case $g(x) \in L^{2}(\Omega)$ : for every $g \in L^{2}(\Omega)$ and any $\epsilon>0$, there is $g^{\epsilon} \in H_{0}^{1}(\Omega)$ which depends on $g$ and $\epsilon$, such that

$$
\left\|g-g^{\epsilon}\right\|<\epsilon .
$$

For the nonlinearity $f$, we take into account two cases: for the weak solutions case, critical exponent $p=4 /(n-2)$; 
for the strong solutions case, critical exponent $p=4 /(n-$ $4)$. Similar to that in $[5,18,19]$, we know that $f$ allows the following decomposition:

$$
f=f_{0}+f_{1} \text {, }
$$

where $f_{0}, f_{1} \in C^{1}(\mathbb{R})$ with $f_{1}(0)=0, f_{2}(0)=0$ and satisfy

$$
\begin{aligned}
& f_{0}(u) u \geqslant 0, \quad \forall u \in \mathbb{R}, \\
& \left|f_{0}^{\prime}(u)\right| \leqslant C\left(1+|u|^{p}\right), \quad \forall u \in \mathbb{R}, \\
& f_{0}^{\prime}(u) \leqslant l_{1}, \quad \forall u \in \mathbb{R}, \\
& \left|f_{1}(u)\right| \leqslant C\left(1+|u|^{\gamma}\right), \quad \forall u \in \mathbb{R}, 0<\gamma<p, \\
& \limsup _{|u| \rightarrow \infty} \frac{f_{1}(u)}{u}>-\lambda_{1}^{\theta} .
\end{aligned}
$$

Set

$$
\sigma=\min \left\{1, \frac{(n+2) \theta-2 n}{n-2}, \frac{2 \theta}{3}, \theta-\frac{(n-2 \theta) \gamma}{2}\right\}
$$

where $\gamma$ is defined by (51).

Analogous to that in [17], we decompose the solution $z(t)=\left(u, u_{t}, \eta^{t}\right)$ of (17) corresponding to initial data $z_{0} \in \mathscr{H}_{1}$ as follows:

$$
z(t)=z_{1}(t)+z_{2}(t)
$$

here $z_{1}(t)=\left(v^{\epsilon}, v_{t}^{\epsilon}, \zeta^{t \epsilon}\right)$ and $z_{2}(t)=\left(w^{\epsilon}, w_{t}^{\epsilon}, \xi^{t \epsilon}\right)$ satisfy the following equations:

$$
\begin{aligned}
& v_{t t}^{\epsilon}+\alpha v_{t}^{\epsilon}+A^{\theta} v^{\epsilon}+\int_{0}^{\infty} \mu(s) A^{\theta} \zeta^{t \epsilon}(s) d s+f_{0}\left(v^{\epsilon}\right)=g \\
& \quad-g^{\epsilon} \\
& \zeta_{t}^{t \epsilon}=-\zeta_{s}^{t \epsilon}+v_{t}^{\epsilon} \\
& \left.v^{\epsilon}(x, t)\right|_{\partial \Omega}=0, \\
& v^{\epsilon}(x, 0)=u_{0}(x), \\
& v_{t}^{\epsilon}(x, 0)=u_{t 0}(x), \\
& \left.\zeta^{t \epsilon}(x, t)\right|_{\partial \Omega}=0, \\
& \zeta^{0 \epsilon}(x, s)=\eta_{0}(x, s),
\end{aligned}
$$

$$
\begin{aligned}
& w_{t t}^{\epsilon}+\alpha w_{t}^{\epsilon}+A^{\theta} w^{\epsilon}+\int_{0}^{\infty} \mu(s) A^{\theta} \xi^{t \epsilon}(s) d s+f(u) \\
& \quad-f_{0}\left(v^{\epsilon}\right)=g^{\epsilon} \\
& \xi_{t}^{t \epsilon}=-\xi_{s}^{t \epsilon}+w_{t}^{\epsilon} \\
& \left.w^{\epsilon}(x, t)\right|_{\partial \Omega}=0 \\
& w^{\epsilon}(x, 0)=0 \\
& w_{t}^{\epsilon}(x, 0)=0 \\
& \left.\xi^{t \epsilon}(x, s)\right|_{\partial \Omega}=0 \\
& \xi^{\theta \epsilon}(x, s)=0
\end{aligned}
$$

According to the general theorem about the existence of global attractors of infinite-dimensional dynamical systems (see $[6,15]$ ), we also need to prove the asymptotic compactness of $\{S(t)\}_{t \geqslant 0}$ in $\mathscr{H}_{1}$.

Similar to the proof of Theorem 5, we can gain the corresponding existence and uniqueness of solutions for (55) and (56); furthermore, we know that the solutions of (55) also form a semigroup. For the sake of convenience, we denote the solution operators of (55) and (56) by $\left\{S_{1}(t)\right\}_{t \geqslant 0}$ and $\left\{S_{2}(t, \cdot)\right\}_{t \geqslant 0}$, respectively. Then, for every $z_{0} \in \mathscr{H}_{1}$, we have

$$
z(t)=S(t) z_{0}=S_{1}(t) z_{0}+S_{2}\left(t, z_{0}\right), \quad \forall t \geqslant 0
$$

Hereafter, we will test the necessary condition of asymptotic smoothness.

At first, akin to the proof of Theorem 6, we can obtain the following result about the solution $z_{1}(t)$ of (55) in $\mathscr{H}_{1}$.

Lemma 7. Assume that $f_{0}$ satisfy (48) and (49), $g \in H^{-1}(\Omega)$, and (6)-(8) hold. Then, for any $\varepsilon_{0}>0$, there is a constant $\epsilon=\epsilon\left(\varepsilon_{0}, g\right)$, such that the solutions of (55) satisfy the following estimates:

$$
\begin{aligned}
\left\|S_{1}(t) z_{0}\right\|_{\mathscr{H}_{1}}^{2} & =\frac{1}{2}\left(\left\|v^{\epsilon}\right\|_{V_{\theta}}^{2}+\left\|v_{t}^{\epsilon}\right\|^{2}+\left\|\zeta^{t \epsilon}\right\|_{\mu, V_{\theta}}^{2}\right) \\
& \leqslant Q\left(\left\|z_{0}\right\|_{\mathscr{H}_{1}}\right) e^{-\beta_{1} t}+\varepsilon_{0}, \quad \forall t \geqslant t_{0},
\end{aligned}
$$

where $Q(\cdot)$ is an increasing function on $[0, \infty)$ and $\beta_{1}$ only depends on $\alpha, \rho$, and $\delta$.

Proof. Taking the $L^{2}$ inner product of (70) with $v_{t}^{\epsilon}+\rho v^{\epsilon}$, we have

$$
\begin{aligned}
\frac{1}{2} \frac{d}{d t} & \left(\left\|v^{\epsilon}\right\|_{V_{\theta}}^{2}+\left\|v_{t}^{\epsilon}+\rho v^{\epsilon}\right\|^{2}+\left\|\zeta^{t \epsilon}\right\|_{\mu, V_{\theta}}^{2}\right)+\rho\left\|v^{\epsilon}\right\|_{V_{\theta}}^{2} \\
& +(\alpha-\rho)\left\|v_{t}^{\epsilon}+\rho v^{\epsilon}\right\|^{2}-\rho(\alpha-\rho)\left\langle v^{\epsilon}, v_{t}^{\epsilon}+\rho v^{\epsilon}\right\rangle \\
& +\left\langle\zeta^{t \epsilon}, \zeta_{s}^{t \epsilon}\right\rangle_{\mu, V_{\theta}}+\rho\left\langle\zeta^{t \epsilon}, v^{\epsilon}\right\rangle_{\mu, V_{\theta}} \\
= & -\left\langle f_{0}\left(v^{\epsilon}\right), v_{t}^{\epsilon}+\rho v^{\epsilon}\right\rangle+\left\langle g-g^{\epsilon}, v_{t}^{\epsilon}+\rho v^{\epsilon}\right\rangle .
\end{aligned}
$$


For the forcing term, we know

$$
\begin{aligned}
-\left\langle g-g^{\epsilon}, v_{t}^{\epsilon}+\rho v^{\epsilon}\right\rangle= & -\frac{d}{d t}\left\langle g-g^{\epsilon}, v^{\epsilon}\right\rangle \\
& -\rho\left\langle g-g^{\epsilon}, v^{\epsilon}\right\rangle, \\
\left\langle g-g^{\epsilon}, v^{\epsilon}\right\rangle \leqslant & \frac{2}{\lambda_{1}^{\theta-1}}\left\|g-g^{\epsilon}\right\|_{H^{-1}(\Omega)}^{2} \\
& +\frac{1}{8}\left\|v^{\epsilon}\right\|_{V_{\theta}}^{2} .
\end{aligned}
$$

About the nonlinearity, from (48), we have

$$
\begin{aligned}
-\left\langle f_{0}\left(v^{\epsilon}\right), v_{t}^{\epsilon}+\rho v^{\epsilon}\right\rangle= & -\frac{d}{d t} \int_{\Omega} F_{0}\left(v^{\epsilon}\right) d x \\
& -\rho\left\langle f_{0}\left(v^{\epsilon}\right), v^{\epsilon}\right\rangle \\
\leqslant & -\frac{d}{d t} \int_{\Omega} F_{0}\left(v^{\epsilon}\right) d x
\end{aligned}
$$

where $F_{0}\left(v^{\epsilon}\right)=\int_{0}^{v^{\epsilon}} f(r) d r$.

In view of (49), (13), (14), and (44), we know

$$
\begin{aligned}
\rho^{2} \int_{\Omega} F_{0}\left(v^{\epsilon}\right) d x \leqslant & \rho^{2} C \int_{\Omega}\left(\left|v^{\epsilon}\right|^{2}+\left|v^{\epsilon}\right|^{2 n /(n-2)}\right) d x \\
\leqslant & \rho^{2} C\left(\left\|v^{\epsilon}\right\|^{2}+\left\|\nabla v^{\epsilon}\right\|^{2}\right) \\
\leqslant & \rho^{2}\left(C+C_{\epsilon}\right)\left\|v^{\epsilon}\right\|^{2} \\
& +\rho^{2} C \epsilon\left\|A^{\theta / 2} v^{\epsilon}\right\|^{2} \\
& \leqslant \rho^{2} C_{\rho, \epsilon, \mu_{0}}\left\|A^{\theta / 2} v^{\epsilon}\right\|^{2}
\end{aligned}
$$

here the constant $C_{\rho, \epsilon, \mu_{0}}$ being very small depends not only on $\epsilon$ and $\rho$, but also on the bound $\mu_{0}$ in (44). Choosing $\rho$ to be sufficiently small, we have

$$
\begin{aligned}
& \rho\left\|v^{\epsilon}\right\|_{V_{\theta}}^{2}+(\alpha-\rho)\left\|v_{t}^{\epsilon}+\rho v^{\epsilon}\right\|^{2} \\
& -\rho(\alpha-\rho)\left\langle v^{\epsilon}, v_{t}^{\epsilon}+\rho v^{\epsilon}\right\rangle \geqslant \rho\left(1-\frac{\alpha \rho}{\lambda_{1}^{\theta}}\right)\left\|v^{\epsilon}\right\|_{V_{\theta}}^{2} \\
& +\left(\frac{1}{2} \alpha-\rho\right)\left\|v_{t}^{\epsilon}+\rho v^{\epsilon}\right\|^{2} \geqslant \frac{\rho}{2}\left\|v^{\epsilon}\right\|_{V_{\theta}}^{2} \\
& +\frac{\alpha}{4}\left\|v_{t}^{\epsilon}+\rho v^{\epsilon}\right\|^{2} .
\end{aligned}
$$

Thanks to Lemma 1 and using (7), we get that

$$
\begin{aligned}
& \left\langle\zeta^{t \epsilon}, \zeta_{s}^{t \epsilon}\right\rangle_{\mu, V_{\theta}} \geqslant \frac{\delta}{2}\left\|\zeta^{t \epsilon}\right\|_{\mu, V_{\theta}}^{2}, \\
& \rho\left\langle\zeta^{t \epsilon}, v^{\epsilon}\right\rangle_{\mu, V_{\theta}} \\
& \geqslant-\rho \int_{\Omega}\left(\int_{0}^{\infty} \mu(s)\left|A^{\theta / 2} \zeta^{t \epsilon}(s)\right|^{2} d s\right)^{1 / 2}
\end{aligned}
$$

$$
\begin{aligned}
& \cdot\left(\int_{0}^{\infty} \mu(s)\left|A^{\theta / 2} v^{\epsilon}\right|^{2} d s\right)^{1 / 2} d x \geqslant-\frac{\delta}{4}\left\|\zeta^{t \epsilon}\right\|_{\mu, V_{\theta}}^{2} \\
& -\frac{k_{0} \rho^{2}}{\delta}\left\|v^{\epsilon}\right\|_{V_{\theta}}^{2} .
\end{aligned}
$$

Then,

$$
\begin{aligned}
& \frac{1}{2} \frac{d}{d t}\left(\left\|v^{\epsilon}\right\|_{V_{\theta}}^{2}+\left\|v_{t}^{\epsilon}+\rho v^{\epsilon}\right\|^{2}+\left\|\zeta^{t \epsilon}\right\|_{\mu, V_{\theta}}^{2}\right. \\
& \left.\quad+2 \int_{\Omega} F_{0}\left(v^{\epsilon}\right) d x-2\left\langle g-g^{\epsilon}, v^{\epsilon}\right\rangle+\check{C}\right)+\left(\frac{\rho}{2}\right. \\
& \left.\quad-\frac{k_{0} \rho^{2}}{\delta}-\rho^{2} C_{\rho, \epsilon, \mu_{0}}\right)\left\|v^{\epsilon}\right\|_{V_{\theta}}^{2}+\frac{\alpha}{4}\left\|v_{t}^{\epsilon}+\rho v^{\epsilon}\right\|_{V_{\theta}}^{2} \\
& +\frac{\delta}{4}\left\|\zeta^{t \epsilon}\right\|_{\mu, V_{2 \theta}}^{2}+\rho^{2} \int_{\Omega} F_{0}\left(v^{\epsilon}\right) d x-\rho\langle g \\
& \left.-g^{\epsilon}, A^{\theta} v^{\epsilon}\right\rangle+\rho \check{C} \leqslant \rho \check{C} ;
\end{aligned}
$$

here, $\check{C}=\left(4 / \lambda_{1}^{\theta-1}\right)\left\|g-g^{\epsilon}\right\|_{H^{-1}(\Omega)}^{2}$.

Obviously, we see that

$$
\frac{\rho}{2}-\frac{k_{0} \rho^{2}}{\delta}-\rho^{2} C_{\rho, \epsilon, \mu_{0}} \geqslant \frac{\rho}{4},
$$

so

$$
\left(\frac{\rho}{2}-\frac{k_{0} \rho^{2}}{\delta}-\rho^{2} C_{\rho, \epsilon, \mu_{0}}\right)\left\|v^{\epsilon}\right\|_{V_{2 \theta}}^{2} \geqslant \frac{\rho}{4}\left\|v^{\epsilon}\right\|_{V_{2 \theta}}^{2} .
$$
that

Then, combining the above estimates with (59), we see

$$
\begin{aligned}
& \frac{1}{2} \frac{d}{d t}\left(\left\|v^{\epsilon}\right\|_{V_{\theta}}^{2}+\left\|v_{t}^{\epsilon}+\rho v^{\epsilon}\right\|^{2}+\left\|\zeta^{t \epsilon}\right\|_{\mu, V_{\theta}}^{2}\right. \\
& \left.\quad+2 \int_{\Omega} F_{0}\left(v^{\epsilon}\right) d x-2\left\langle g-g^{\epsilon}, v^{\epsilon}\right\rangle+\check{C}\right) \\
& \quad+\frac{\beta_{1}}{2}\left(\left\|v^{\epsilon}\right\|_{V_{\theta}}^{2}+\left\|v_{t}^{\epsilon}+\rho v^{\epsilon}\right\|^{2}+\left\|\zeta^{t \epsilon}\right\|_{\mu, V_{\theta}}^{2}\right. \\
& \left.+2 \int_{\Omega} F_{0}\left(v^{\epsilon}\right) d x-2\left\langle g-g^{\epsilon}, v^{\epsilon}\right\rangle+\check{C}\right) \leqslant \rho \check{C} \\
& \quad<\frac{4 \rho}{\lambda_{1}^{\theta-1}} \epsilon^{2},
\end{aligned}
$$

where $\beta_{1}=\min \left\{\rho / 2, \alpha / 2, \delta / 2, \rho^{2}\right\}$.

Obviously, there exist constants $\check{C}_{1}, \check{C}_{2}$, such that

$$
\begin{aligned}
& \check{C}_{1}\left\|z_{1}(t)\right\|_{\mathscr{H}_{1}}^{2} \leqslant \frac{1}{2}\left(\left\|v^{\epsilon}\right\|_{V_{\theta}}^{2}+\left\|v_{t}^{\epsilon}+\rho v^{\epsilon}\right\|^{2}+\left\|\zeta^{t \epsilon}\right\|_{\mu, V_{\theta}}^{2}\right. \\
& \left.\quad+2 \int_{\Omega} F_{0}\left(v^{\epsilon}\right) d x-2\left\langle g-g^{\epsilon}, v^{\epsilon}\right\rangle+\check{C}\right) \\
& \quad \leqslant \check{C}_{2}\left\|z_{1}(t)\right\|_{\mathscr{H}_{1}}^{2} ;
\end{aligned}
$$


thus, using Gronwall lemma and taking $4 \rho \epsilon^{2} / \lambda_{1}^{\theta-1} \beta_{1} \check{C}_{1}<\varepsilon_{0}$, as $t \geqslant t_{0}$, we obtain

$$
\begin{aligned}
\left\|z_{1}(t)\right\|_{\mathscr{H}_{1}}^{2} & \leqslant \frac{\check{C}_{2}}{\check{C}_{1}}\|z(0)\|_{\mathscr{H}_{1}}^{2} e^{-\beta_{1} t}+\frac{4 \rho \epsilon^{2}}{\lambda_{1}^{\theta-1} \beta_{1} \check{C}_{1}} \\
& <Q\left(\left\|z_{0}\right\|_{\mathscr{H}_{1}}\right) e^{-\beta_{1} t}+\varepsilon_{0} .
\end{aligned}
$$

The proof is complete.

Lemma 8. Let the nonlinearity $f$ satisfy (2)-(3) and (51)-(52), $g \in H^{-1}(\Omega)$, and (7)-(9) hold. Then, for every given $T>0$ and $\epsilon>0$, there is a position constant

$$
N_{0}=N_{0}\left(T,\|g\|_{H^{-1}(\Omega)},\left\|z_{0}\right\|_{\mathscr{H}_{1}}, \delta\right),
$$

such that the solutions of (56) satisfy

$$
\begin{aligned}
& \left\|S_{2}\left(T, z_{0}\right)\right\|_{\mathscr{P}_{1+\sigma}}^{2} \\
& \quad=\frac{1}{2}\left(\left\|w^{\epsilon}\right\|_{V_{\theta+\sigma}}^{2}+\left\|w_{t}^{\epsilon}\right\|_{V_{\sigma}}^{2}+\left\|\xi^{T e}\right\|_{\mu, V_{\theta+\sigma}}^{2}\right) \leqslant N_{0},
\end{aligned}
$$

where $\sigma=\min \{1,((n+2) \theta-2 n) /(n-2), 2 \theta / 3, \theta-(n-2 \theta) \gamma / 2\}$.

Proof. Multiplying (56) by $A^{\sigma}\left(w_{t}^{\epsilon}+\rho w^{\epsilon}\right)$, we get that

$$
\begin{aligned}
\frac{1}{2} \frac{d}{d t} & \left(\left\|w^{\epsilon}\right\|_{V_{\theta+\sigma}}^{2}+\left\|w_{t}^{\epsilon}+\rho w^{\epsilon}\right\|_{V_{\sigma}}^{2}+\left\|\xi^{\epsilon \epsilon}\right\|_{\mu, V_{\theta+\sigma}}^{2}\right) \\
& +\rho\left\|w^{\epsilon}\right\|_{V_{\theta+\sigma}}^{2}+(\alpha-\rho)\left\|w_{t}^{\epsilon}+\rho w\right\|_{V_{\sigma}}^{2} \\
& -\rho(\alpha-\rho)\left\langle w^{\epsilon}, w_{t}^{\epsilon}+\rho w^{\epsilon}\right\rangle_{V_{\sigma}}+\left\langle\xi^{t \epsilon}, \xi_{s}^{t \epsilon}\right\rangle_{\mu, V_{\theta+\sigma}} \\
& +\rho\left\langle\xi^{t \epsilon}, w^{\epsilon}\right\rangle_{\mu, V_{\theta+\sigma}} \\
= & -\left\langle f(u)-f_{0}\left(v^{\epsilon}\right), A^{\sigma}\left(w_{t}^{\epsilon}+\rho w^{\epsilon}\right)\right\rangle \\
& +\left\langle g^{\epsilon}, A^{\sigma}\left(w_{t}^{\epsilon}+\rho w^{\epsilon}\right)\right\rangle .
\end{aligned}
$$

Using Lemma 1, we can obtain

$$
\begin{aligned}
& \left\langle\xi^{t^{\epsilon}}, \xi_{s}^{t \epsilon}\right\rangle_{\mu, V_{\theta+\sigma}} \geqslant \frac{\delta}{2}\left\|\xi^{t \epsilon}\right\|_{\mu, V_{\theta+\sigma}}^{2}, \\
& \rho\left\langle\xi^{t \epsilon}, w^{\epsilon}\right\rangle_{\mu, V_{\theta+\sigma}} \\
& \geqslant-\rho \int_{\Omega} \int_{0}^{\infty} \mu(s) A^{(\theta+\sigma) / 2} \xi^{t \epsilon} A^{(\theta+\sigma) / 2} w^{\epsilon} d s d x \\
& \geqslant-\rho \int_{\Omega}\left(\int_{0}^{\infty} \mu(s)\left|A^{(\theta+\sigma) / 2} \xi^{t \epsilon}\right|^{2} d s\right)^{1 / 2} \\
& \cdot\left(\int_{0}^{\infty} \mu(s)\left|A^{(\theta+\sigma) / 2} w^{\epsilon}\right|^{2} d s\right)^{1 / 2} d x \\
& \geqslant-\frac{\delta}{4}\left\|\xi^{t \epsilon}\right\|_{\mu, V_{\theta+\sigma}}^{2}-\frac{k_{0} \rho^{2}}{\delta}\left\|w^{\epsilon}\right\|_{V_{\theta+\sigma}}^{2} .
\end{aligned}
$$

Similarly, we have

$$
\begin{aligned}
\frac{1}{2} \frac{d}{d t}( & \left.\left\|w^{\epsilon}\right\|_{V_{\theta+\sigma}}^{2}+\left\|w_{t}^{\epsilon}+\rho w^{\epsilon}\right\|_{V_{\sigma}}^{2}+\left\|\xi^{t \epsilon}\right\|_{\mu, V_{\theta+\sigma}}^{2}\right) \\
& +\left(\rho-\frac{k_{0} \rho^{2}}{\delta}\right)\left\|w^{\epsilon}\right\|_{V_{\theta+\sigma}}^{2}+(\alpha-\rho)\left\|w_{t}^{\epsilon}+\rho w^{\epsilon}\right\|_{V_{\sigma}}^{2} \\
& -\rho(\alpha-\rho)\left\langle w^{\epsilon}, w_{t}^{\epsilon}+\rho w^{\epsilon}\right\rangle_{V_{\sigma}}+\frac{\delta}{4}\left\|\xi^{\epsilon \epsilon}\right\|_{\mu, V_{\theta+\sigma}}^{2} \\
= & -\left\langle f(u)-f_{0}\left(v^{\epsilon}\right), A^{\sigma}\left(w_{t}^{\epsilon}+\rho w^{\epsilon}\right)\right\rangle \\
& +\left\langle g^{\epsilon}, A^{\sigma}\left(w_{t}^{\epsilon}+\rho w^{\epsilon}\right)\right\rangle .
\end{aligned}
$$

Choosing $\rho$ to be sufficiently small, such that $\alpha / 2-\rho \geqslant \alpha / 4$ and $1-\rho \alpha / \lambda_{1}^{\theta}-k_{0} \rho / \delta \geqslant 1 / 2$, we can obtain that

$$
\begin{aligned}
& \rho\left(1-\frac{k_{0} \rho}{\delta}\right)\left\|w^{\epsilon}\right\|_{V_{\theta+\sigma}}^{2}+(\alpha-\rho)\left\|w_{t}^{\epsilon}+\rho w^{\epsilon}\right\|_{V_{\sigma}}^{2} \\
& -\rho(\alpha-\rho)\left\langle w^{\epsilon}, w_{t}^{\epsilon}+\rho w^{\epsilon}\right\rangle_{V_{\sigma}} \\
& \geqslant \rho\left(1-\frac{k_{0} \rho}{\delta}\right)\left\|w^{\epsilon}\right\|_{V_{\theta+\sigma}}^{2}+(\alpha-\rho)\left\|w_{t}^{\epsilon}+\rho w^{\epsilon}\right\|_{V_{\sigma}}^{2} \\
& -\rho \alpha\left\langle w^{\epsilon}, w_{t}^{\epsilon}+\rho w^{\epsilon}\right\rangle_{V_{\sigma}}+\rho^{2}\left\langle w^{\epsilon}, w_{t}^{\epsilon}\right\rangle_{V_{\sigma}} \\
& \geqslant \rho\left(1-\frac{k_{0} \rho}{\delta}\right)\left\|w^{\epsilon}\right\|_{V_{\theta+\sigma}}^{2}+(\alpha-\rho)\left\|w_{t}^{\epsilon}+\rho w^{\epsilon}\right\|_{V_{\sigma}}^{2} \\
& -\frac{\alpha \rho^{2}}{\lambda_{1}^{\theta}}\left\|w^{\epsilon}\right\|_{V_{\theta+\sigma}}^{2}-(\alpha-\rho)\left\langle\rho w^{\epsilon}, w_{t}^{\epsilon}\right\rangle_{V_{\sigma}} \\
& \geqslant \rho\left(1-\frac{k_{0} \rho}{\delta}\right)\left\|w^{\epsilon}\right\|_{V_{\theta+\sigma}}^{2}+(\alpha-\rho)\left\|w_{t}^{\epsilon}+\rho w^{\epsilon}\right\|_{V_{\sigma}}^{2} \\
& -\frac{\alpha \rho^{2}}{\lambda_{1}^{\theta}}\left\|w^{\epsilon}\right\|_{V_{\theta+\sigma}}^{2}-\frac{\alpha}{2}\left\|w_{t}^{\epsilon}+\rho w^{\epsilon}\right\|_{V_{\sigma}}^{2} \geqslant \frac{\rho}{2}\left\|w^{\epsilon}\right\|_{V_{\theta+\sigma}}^{2} \\
& +\frac{\alpha}{4}\left\|w_{t}^{\epsilon}+\rho w^{\epsilon}\right\|_{V_{\sigma}}^{2} .
\end{aligned}
$$

Since $f=f_{0}+f_{1}$, we have

$$
\begin{gathered}
\left\langle f(u)-f_{0}\left(v^{\epsilon}\right), A^{\sigma}\left(w_{t}^{\epsilon}+\rho w^{\epsilon}\right)\right\rangle \\
=\left\langle f(u)-f\left(v^{\epsilon}\right), A^{\sigma}\left(w_{t}^{\epsilon}+\rho w^{\epsilon}\right)\right\rangle \\
\quad+\left\langle f_{1}\left(v^{\epsilon}\right), A^{\sigma}\left(w_{t}^{\epsilon}+\rho w^{\epsilon}\right)\right\rangle .
\end{gathered}
$$

We deal with the terms in (77) one by one.

First, by virtue of (3), we deduce that

$$
\begin{aligned}
- & \left\langle f(u)-f\left(v^{\epsilon}\right), A^{\sigma}\left(w_{t}^{\epsilon}+\rho w^{\epsilon}\right)\right\rangle \\
\leqslant & \mid \int_{\Omega}\left(f^{\prime}(u) A^{3 \sigma / 4} u-f^{\prime}\left(v^{\epsilon}\right) A^{3 \sigma / 4} v^{\epsilon}\right) \\
& \cdot A^{\sigma / 4}\left(w_{t}^{\epsilon}+\rho w^{\epsilon}\right) d x \mid \leqslant C \int_{\Omega}\left(1+|u|^{4 /(n-2)}\right. \\
& \left.+\left|v^{\epsilon}\right|^{4 /(n-2)}\right)\left|A^{3 \sigma / 4} w^{\epsilon}\right|\left|A^{\sigma / 4}\left(w_{t}^{\epsilon}+\rho w^{\epsilon}\right)\right| d x
\end{aligned}
$$


Mathematical Problems in Engineering

9

$$
\begin{aligned}
& +2 C \int_{\Omega}\left(1+|u|^{4 /(n-2)}+\left|v^{\epsilon}\right|^{4 /(n-2)}\right)\left|A^{3 \sigma / 4} v^{\epsilon}\right| \\
& \cdot\left|A^{\sigma / 4}\left(w_{t}^{\epsilon}+\rho w^{\epsilon}\right)\right| d x .
\end{aligned}
$$

Second, since $\theta \in[2 n /(n+2), n / 2)$, we have

$$
\begin{aligned}
& C \int_{\Omega}\left(1+|u|^{4 /(n-2)}+\left|v^{\epsilon}\right|^{4 /(n-2)}\right)\left|A^{3 \sigma / 4} w^{\epsilon}\right| \\
& \cdot\left|A^{\sigma / 4}\left(w_{t}^{\epsilon}+\rho w^{\epsilon}\right)\right| d x \\
& \leqslant C\left(\int_{\Omega}\left(1+|u|^{4 /(n-2)}+\left|v^{\epsilon}\right|^{4 /(n-2)}\right)^{n / \theta} d x\right)^{\theta / n} \\
& \cdot\left(\int_{\Omega}\left|A^{3 \sigma / 4} w^{\epsilon}\right|^{2 n /(n-2(\theta-\sigma / 2))} d x\right)^{(n-2(\theta-\sigma / 2)) / 2 n} \\
& \cdot\left(\int_{\Omega}\left|A^{\sigma / 4}\left(w_{t}^{\epsilon}+\rho w^{\epsilon}\right)\right|^{2 n /(n-\sigma)} d x\right)^{(n-\sigma) / 2 n} \leqslant C(1 \\
& \left.+\|u\|_{L^{4 n /(n-2) \theta}}^{4 /(n-2)}+\left\|v^{\epsilon}\right\|_{L^{4 n /(n-2) \theta}}^{4 /(n-2)}\right)\left\|A^{3 \sigma / 4} w^{\epsilon}\right\|_{L^{2 n /(n-2(\theta-\sigma / 2))}} \\
& \cdot\left\|A^{\sigma / 4}\left(w_{t}^{\epsilon}+\rho w^{\epsilon}\right)\right\|_{L^{2 n /(n-\sigma)}} \leqslant C\left(1+\|u\|_{L^{2 n /(n-2 \theta)}}^{4 /(n-2)}\right. \\
& \left.+\left\|v^{\epsilon}\right\|_{L^{2 n /(n-2 \theta)}}^{4 /(n-2)}\right)\left\|A^{3 \sigma / 4} w^{\epsilon}\right\|_{L^{2 n /(n-2(\theta-\sigma / 2))}} \\
& \cdot\left\|A^{\sigma / 4}\left(w_{t}^{\epsilon}+\rho w^{\epsilon}\right)\right\|_{L^{2 n /(n-\sigma)}} \leqslant C(1 \\
& \left.+\left\|A^{\theta / 2} u\right\|^{4 /(n-2)}+\left\|A^{\theta / 2} v^{\epsilon}\right\|^{4 /(n-2)}\right)\left\|A^{(\theta+\sigma) / 2} w^{\epsilon}\right\| \\
& \cdot\left\|A^{\sigma / 2}\left(w_{t}^{\epsilon}+\rho w^{\epsilon}\right)\right\| \leqslant C_{\mu_{0}, \varepsilon_{0}}\left\|A^{(\theta+\sigma) / 2} w^{\epsilon}\right\|^{2} \\
& +\frac{\alpha}{4}\left\|A^{\sigma / 2}\left(w_{t}^{\epsilon}+\rho w^{\epsilon}\right)\right\|^{2} \text {, }
\end{aligned}
$$

where $4 n /(n-2) \theta \leqslant 2 n /(n-2 \theta)$, and we have used the embedding $V_{\theta}=D\left(A^{\theta / 2}\right) \hookrightarrow L^{2 n /(n-2 \theta)} \hookrightarrow L^{4 n /(n-2) \theta}$, $V_{\sigma / 2}=D\left(A^{\sigma / 4}\right) \hookrightarrow L^{2 n /(n-\sigma)}, V_{\theta-\sigma / 2}=D\left(A^{(2 \theta-\sigma) / 4}\right) \hookrightarrow$ $L^{2 n /(n-2(\theta-\sigma / 2))}$.

Third, from (53), we obtain that

$$
\begin{aligned}
& 2 C \int_{\Omega}\left(1+|u|^{4 /(n-2)}+\left|v^{\epsilon}\right|^{4 /(n-2)}\right)\left|A^{3 \sigma / 4} v^{\epsilon}\right| \mid A^{\sigma / 4}\left(w_{t}^{\epsilon}\right. \\
& \left.+\rho w^{\epsilon}\right) \mid d x \leqslant 2 C\left(\int _ { \Omega } \left(1+|u|^{4 /(n-2)}\right.\right. \\
& \left.\left.\quad+\left|v^{\epsilon}\right|^{4 /(n-2)}\right)^{n /(\theta-\sigma)} d x\right)^{(\theta-\sigma) / n} \\
& \cdot\left(\int_{\Omega}\left|A^{3 \sigma / 4} v^{\epsilon}\right|^{2 n /(n-2(\theta-3 \sigma / 2))} d x\right)^{(n-2(\theta-3 \sigma / 2)) / 2 n} \\
& \quad \cdot\left(\int_{\Omega}\left|A^{\sigma / 4}\left(w_{t}^{\epsilon}+\rho w^{\epsilon}\right)\right|^{2 n /(n-\sigma)} d x\right)^{(n-\sigma) / 2 n}
\end{aligned}
$$

$$
\begin{aligned}
& \leqslant 2 C\left(1+\|u\|_{L^{4 n /(n-2)(\theta-\sigma)}}^{4 /(n-2)}+\left\|v^{\epsilon}\right\|_{L^{4 n /(n-2)(\theta-\sigma)}}^{4 /(n-2)}\right) \\
& \cdot\left\|A^{3 \sigma / 4} v^{\epsilon}\right\|_{L^{2 n /(n-2(\theta-3 \sigma / 2))}}\left\|A^{\sigma / 4}\left(w_{t}^{\epsilon}+\rho w^{\epsilon}\right)\right\|_{L^{2 n /(n-\sigma)}} \\
& \leqslant 2 C\left(1+\|u\|_{L^{2 n /(n-2 \theta)}}^{4 /(n-2)}+\left\|v^{\epsilon}\right\|_{L^{2 n /(n-2 \theta)}}^{4 /(n-2)}\right) \\
& \cdot\left\|A^{3 \sigma / 4} v^{\epsilon}\right\|_{L^{2 n /(n-2(\theta-\sigma / 2))}}\left\|A^{\sigma / 4}\left(w_{t}^{\epsilon}+\rho w^{\epsilon}\right)\right\|_{L^{2 n /(n-\sigma)}} \\
& \leqslant 2 C\left(1+\left\|A^{\theta / 2} u\right\|^{4 /(n-2)}+\left\|A^{\theta / 2} v^{\epsilon}\right\|^{4 /(n-2)}\right) \\
& \cdot\left\|A^{\theta / 2} v^{\epsilon}\right\| \cdot\left\|A^{\sigma / 2}\left(w_{t}^{\epsilon}+\rho w^{\epsilon}\right)\right\| \leqslant 2 C(1 \\
& \left.+\left\|A^{\theta / 2} u\right\|^{4 /(n-2)}+\left\|A^{\theta / 2} v^{\epsilon}\right\|^{4 /(n-2)}\right)^{2}\left\|A^{\theta / 2} v^{\epsilon}\right\|^{2} \\
& +\frac{\alpha}{4}\left\|A^{\sigma / 2}\left(w_{t}^{\epsilon}+\rho w^{\epsilon}\right)\right\|^{2} \leqslant C_{\mu_{0}, \varepsilon_{0}}+\frac{\alpha}{4} \| A^{\sigma / 2}\left(w_{t}^{\epsilon}\right. \\
& \left.+\rho w^{\epsilon}\right) \|^{2},
\end{aligned}
$$

where $4 n /(n-2)(\theta-\sigma) \leqslant 2 n /(n-2 \theta)$, and we have used the embedding $V_{\theta}=D\left(A^{\theta / 2}\right) \hookrightarrow L^{2 n /(n-2 \theta)} \hookrightarrow L^{4 n /(n-2)(\theta-\sigma)}$, $V_{\sigma / 2}=D\left(A^{\sigma / 4}\right) \hookrightarrow L^{2 n /(n-\sigma)}, V_{\theta-3 \sigma / 2}=D\left(A^{(2 \theta-3 \sigma) / 4}\right) \hookrightarrow$ $L^{2 n /(n-2(\theta-3 \sigma / 2))}$.

Finally, by virtue of (51), we get

$$
\begin{aligned}
- & \left\langle f_{1}\left(v^{\epsilon}\right), A^{\sigma}\left(w_{t}^{\epsilon}+\rho w^{\epsilon}\right)\right\rangle \\
& =-\int_{\Omega} f_{1}^{\prime}\left(v^{\epsilon}\right) \cdot A^{3 \sigma / 4} v^{\epsilon} \cdot A^{\sigma / 4}\left(w_{t}^{\epsilon}+\rho w^{\epsilon}\right) d x \\
& \leqslant C \int_{\Omega}\left(1+\left|v^{\epsilon}\right|^{\gamma}\right)\left|A^{3 \sigma / 4} v^{\epsilon}\right|\left|A^{\sigma / 4}\left(w_{t}^{\epsilon}+\rho w^{\epsilon}\right)\right| d x \\
& \leqslant C\left(\int_{\Omega}\left(1+\left|v^{\epsilon}\right|^{\gamma}\right)^{n /(\theta-\sigma)} d x\right)^{(\theta-\sigma) / n} \\
& \cdot\left(\int_{\Omega}\left|A^{3 \sigma / 4} v^{\epsilon}\right|^{2 n /(n-2(\theta-3 \sigma / 2))} d x\right)^{(n-2(\theta-3 \sigma / 2)) / 2 n} \\
& \cdot\left(\int_{\Omega}\left|A^{\sigma / 4}\left(w_{t}^{\epsilon}+\rho w^{\epsilon}\right)\right|^{2 n /(n-\sigma)} d x\right)^{(n-\sigma) / 2 n} \\
& \leqslant C\left(1+\left\|v^{\epsilon}\right\|_{L^{n \gamma /(\theta-\sigma)}}^{\gamma}\right)\left\|A^{3 \sigma / 4} v^{\epsilon}\right\|_{L^{2 n /(n-2(\theta-3 \sigma / 2))}} \\
& \cdot\left\|A^{\sigma / 4}\left(w_{t}^{\epsilon}+\rho w^{\epsilon}\right)\right\|_{L^{2 n /(n-\sigma)}} \leqslant C\left(1+\left\|v^{\epsilon}\right\|_{L^{2 n /(n-2 \theta)}}^{\gamma}\right) \\
& \cdot\left\|A^{3 \sigma / 4} v^{\epsilon}\right\|_{L^{2 n /(n-2(\theta-3 \sigma / 2))}}\left\|A^{\sigma / 4}\left(w_{t}^{\epsilon}+\rho w^{\epsilon}\right)\right\|_{L^{2 n /(n-\sigma)}} \\
& \leqslant \frac{\alpha}{4}\left\|A^{\sigma / 2}\left(w_{t}^{\epsilon}+\rho w^{\epsilon / 2}\right)\right\|^{2} \leqslant C_{\varepsilon_{0}} \\
& \leqslant C\left(1+\left\|A^{\theta / 2} v^{\epsilon}\right\|^{\gamma}\right)\left\|A^{\theta / 2} v^{\epsilon}\right\|\left\|A^{\sigma / 2}\left(w_{t}^{\epsilon}+\rho w^{\epsilon}\right)\right\|
\end{aligned}
$$

(81) 
where we have employed the inequality $\sigma \leqslant \theta-(n-$ $2 \theta) \gamma / 2, n \gamma /(\theta-\sigma) \leqslant 2 n /(n-2 \theta)$ and used the embedding $V_{\theta} \hookrightarrow L^{2 n /(n-2 \theta)} \hookrightarrow L^{n \gamma /(\theta-\sigma)}, V_{\sigma / 2}=D\left(A^{\sigma / 4}\right) \hookrightarrow L^{2 n /(n-\sigma)}$ and $V_{\theta-3 \sigma / 2}=D\left(A^{(2 \theta-3 \sigma) / 4}\right) \hookrightarrow L^{2 n /(n-2(\theta-3 \sigma / 2))}$.

Obviously, we see

$$
\begin{aligned}
\left\langle g^{\epsilon}, A^{\sigma}\left(w_{t}^{\epsilon}+\rho w^{\epsilon}\right)\right\rangle= & \frac{d}{d t}\left\langle g^{\epsilon}, A^{\sigma} w^{\epsilon}\right\rangle \\
& +\rho\left\langle g^{\epsilon}, A^{\sigma} w^{\epsilon}\right\rangle,
\end{aligned}
$$

and, noting that $0<\sigma<2 \theta / 3$, we know that

$$
\begin{aligned}
\left\langle g^{\epsilon}, A^{\sigma} w^{\epsilon}\right\rangle & \leqslant\left\|g^{\epsilon}\right\|\left\|A^{\sigma} w^{\epsilon}\right\| \\
& \leqslant \frac{1}{2 \lambda_{1}^{\theta-\sigma}}\left\|g^{\epsilon}\right\|^{2}+\frac{\lambda_{1}^{\theta-\sigma}}{2}\left\|A^{\sigma} w^{\epsilon}\right\|^{2} \\
& \leqslant \frac{1}{2 \lambda_{1}^{\theta-\sigma}}\left\|g^{\epsilon}\right\|^{2}+\frac{1}{2}\left\|A^{(\theta+\sigma) / 2} w^{\epsilon}\right\|^{2} .
\end{aligned}
$$

Combining above estimates, we can deduce that

$$
\begin{aligned}
& \frac{1}{2} \frac{d}{d t}\left(\left\|w^{\epsilon}\right\|_{V_{\theta+\sigma}}^{2}+\left\|w_{t}^{\epsilon}+\rho w^{\epsilon}\right\|_{V_{\sigma}}^{2}+\left\|\xi^{\epsilon}\right\|_{\mu, V_{\theta+\sigma}}^{2}\right. \\
& \left.-2\left\langle g^{\epsilon}, A^{\sigma} w^{\epsilon}\right\rangle+\breve{C}\right) \leqslant \frac{\beta_{2}}{2}\left(\left\|w^{\epsilon}\right\|_{V_{\theta+\sigma}}^{2}\right. \\
& \left.\quad+\left\|w_{t}^{\epsilon}+\rho w^{\epsilon}\right\|_{V_{\sigma}}^{2}+\left\|\xi^{t \epsilon}\right\|_{\mu, V_{\theta+\sigma}}^{2}-2\left\langle g^{\epsilon}, A^{\sigma} w^{\epsilon}\right\rangle+\breve{C}\right) \\
& \quad+C
\end{aligned}
$$

where $\breve{C}=\left(1 / \lambda_{1}^{\theta-\sigma}\right)\left\|g^{\epsilon}\right\|^{2}, \beta_{2}=\max \left\{2 C_{\mu_{0}, \varepsilon_{0}}-\rho, \alpha, \delta / 2, \rho\right\}$.

Applying the Gronwall inequality and integrating over $[0, t]$, we gain that

$$
\begin{aligned}
& \frac{1}{2}\left(\left\|w^{\epsilon}(t)\right\|_{V_{\theta+\sigma}}^{2}+\left\|w_{t}^{\epsilon}(t)+\rho w^{\epsilon}(t)\right\|_{V_{\sigma}}^{2}+\left\|\xi^{t \epsilon}(s)\right\|_{\mu, V_{\theta+\sigma}}^{2}\right. \\
& \left.-2\left\langle g^{\epsilon}(x), A^{\sigma} w^{\epsilon}(t)\right\rangle+\breve{C}\right) \leqslant \frac{1}{2}\left(\left\|w^{\epsilon}(0)\right\|_{V_{\theta+\sigma}}^{2}\right. \\
& +\left\|w_{t}^{\epsilon}(0)+\rho w^{\epsilon}(0)\right\|_{V_{\sigma}}^{2}+\left\|\xi^{\epsilon \epsilon}(s)\right\|_{\mu, V_{\theta+\sigma}}^{2} \\
& \left.-2\left\langle g^{\epsilon}(x), A^{\sigma} w^{\epsilon}(0)\right\rangle+\breve{C}\right) e^{\beta_{2} t}+\frac{C}{\beta_{2}}\left(e^{\beta_{2} t}-1\right) \\
& \leqslant\left(\frac{\breve{C}}{2}+\frac{C}{\beta_{2}}\right) e^{\beta_{2} t} .
\end{aligned}
$$

Clearly, there exist constants $\breve{C}_{1}, \breve{C}_{2}$, such that

$$
\begin{aligned}
\breve{C}_{1} & \left\|z_{2}(t)\right\|_{\mathscr{H}_{1+\sigma}}^{2} \leqslant \frac{1}{2}\left(\left\|w^{\epsilon}(t)\right\|_{V_{\theta+\sigma}}^{2}\right. \\
& +\left\|w_{t}^{\epsilon}(t)+\rho w^{\epsilon}(t)\right\|_{V_{\sigma}}^{2}+\left\|\xi^{t \epsilon}(s)\right\|_{\mu, V_{\theta+\sigma}}^{2} \\
& \left.-2\left\langle g^{\epsilon}(x), A^{\sigma} w^{\epsilon}(t)\right\rangle+\breve{C}\right) \leqslant \breve{C}_{2}\left\|z_{2}(t)\right\|_{\mathscr{H}_{1+\sigma}}^{2},
\end{aligned}
$$

so

$$
\left\|z_{2}(t)\right\|_{\mathscr{H}_{1+\sigma}}^{2} \leqslant \frac{1}{\breve{C}_{1}}\left(\frac{\breve{C}}{2}+\frac{C}{\beta_{2}}\right) e^{\beta_{2} t}
$$

That is,

$$
\begin{aligned}
& \left\|S_{2}\left(T, z_{0}\right)\right\|_{\mathscr{H}_{1+\sigma}}^{2} \\
& \quad=\frac{1}{2}\left(\left\|w^{\epsilon}(T)\right\|_{\theta+\sigma}^{2}+\left\|w_{t}(T)\right\|_{V_{\sigma}}^{2}+\left\|\xi^{T e}\right\|_{\mu, V_{\theta+\sigma}}^{2}\right) \\
& \quad \leqslant N_{0},
\end{aligned}
$$

where $N_{0}=N_{0}\left(T, g,\left\|z_{0}\right\|_{\mathscr{H}_{1}}, \alpha, \delta\right)$.

The proof is complete.

In addition, for any $\xi_{0} \in L_{\mu}^{2}\left(\mathbb{R}^{+} ; V_{\theta}\right)$, Cauchy problem (see $[9,15,16])$

$$
\begin{aligned}
& \xi_{t}^{t}=-\xi_{s}^{t}+w_{t}, \quad t>0 \\
& \xi^{0}=\xi_{0}
\end{aligned}
$$

has a unique solution $\xi^{t} \in C\left([0, \infty) ; L_{\mu}^{2}\left(\mathbb{R}^{+} ; V_{\theta}\right)\right)$ and explicit expression:

$$
\xi^{t}(x, s)= \begin{cases}w(x, t)-w(x, t-s), & 0<s \leqslant t \\ w(x, t)-w_{0}, & s>t .\end{cases}
$$

Let $B_{0}^{*}$ be the bounded absorbing set in $\mathscr{H}_{1}$ obtained from Theorem 6; we can obtain the following.

Lemma 9. Set

$$
\mathscr{K}_{T}^{\epsilon}:=\Pi S_{2}\left(T, B_{0}^{*}\right) .
$$

Under the assumption of Lemma 8, for every given $T>0, \forall \epsilon>$ 0 , there is a positive constant $N_{1}=N_{1}\left(T, g, \epsilon,\left\|B_{0}^{*}\right\|_{\mathscr{H}_{1}}, \alpha, \delta\right)$, such that

(i) $\mathscr{K}_{T}^{\epsilon}$ is bounded in $L_{\mu}^{2}\left(\mathbb{R}^{+} ; V_{\theta+\sigma}\right) \cap H_{\mu}^{1}\left(\mathbb{R}^{+} ; H\right)$;

(ii) $\sup _{\xi \in \mathscr{K}_{T}^{\epsilon}}\|\xi(s)\|_{V_{\theta}}^{2} \leqslant N_{1}$,

where $\sigma=\min \{1,((n+2) \theta-2 n) /(n-2), 2 \theta / 3, \theta-(n-2 \theta) \gamma / 2\}$. $S_{2}(t, \cdot)$ is the solution operators of (56), and $\Pi: V_{\theta} \times H \times$ $L_{\mu}^{2}\left(\mathbb{R}^{+} ; V_{\theta}\right) \rightarrow L_{\mu}^{2}\left(\mathbb{R}^{+} ; V_{\theta}\right)$ is a projection operator.

Proof. Due to the explicit expression (90), we can deduce that

$$
\xi_{s}^{t}(x, s)= \begin{cases}w_{s}(x, t-s), & 0<s \leqslant t \\ 0, & s>t,\end{cases}
$$

which, combined with Lemma 8, implies that (i) holds.

After that, we can easily know that

$$
\left\|\xi^{T}(x, s)\right\|_{V_{\theta}} \leqslant \begin{cases}\|w(T)-w(T-s)\|_{V_{\theta}}, & 0<s \leqslant T . \\ \|w(T)\|_{V_{\theta}}, & s>T .\end{cases}
$$

By virtue of (88), we get that (ii) holds.

We complete the proof. 
Moreover, applying Lemma 2, we can obtain that $\mathscr{K}_{T}^{\epsilon}$ is relatively compact in $L_{\mu}^{2}\left(\mathbb{R}^{+} ; V_{\theta}\right)$. Then, using the compact embedding $V_{\theta+\sigma} \hookrightarrow V_{\theta}$ once again, we can get the following.

Lemma 10. Let $S_{2}(t, \cdot)$ be the corresponding solution operator of (56). Under the assumption of Lemma 8, for any $T>0$, $S_{2}\left(T, B_{0}^{*}\right)$ is relatively compact in $\mathscr{H}_{1}$.

By applying the classical theory (e.g., see $[6,20])$, from Lemma 3, Theorem 6, and Lemmas 7 and 10, we can gain the main result.

Theorem 11 (global attractor in $\left.\mathscr{H}_{1}\right)$. Let the nonlinearity $f$ satisfy (2)-(3), $g \in H^{-1}(\Omega)$, (7)-(9) hold, and $\{S(t)\}_{t \geqslant 0}$ be the solution semigroup of (17)-(18) in $\mathscr{H}_{1}$. Then $\{S(t)\}_{t \geqslant 0}$ has a global attractor $\mathscr{A}_{0}$ in $\mathscr{H}_{1}$; namely, $\mathscr{A}_{0}$ is compact and invariant in $\mathscr{H}_{1}$ and attracts every bounded subset of $\mathscr{H}_{1}$ with respect to the $\mathscr{H}_{1}$-norm.

\section{Global Attractors in $\mathscr{H}_{2}$}

4.1. The Existence and Uniqueness of Strong Solution. First of all, we define the concept of strong solution for dynamical systems (17)-(18).

Definition 12. Define $I=[0, T], \forall T>0$. Let $g \in L^{2}(\Omega)$ and $z_{0} \in \mathscr{H}_{2}$. A ternary form $z=\left(u, u_{t}, \eta^{t}\right)$ is said to be the strong solution of problem (17)-(18) in the time interval $I$ with initial data $z(0)=z_{0}$, if $z$ satisfies (27), and

$$
\begin{aligned}
u & \in C\left(I ; V_{2 \theta}\right), \\
u_{\mathrm{t}} & \in L^{2}\left(I ; V_{2 \theta}\right), \\
\eta^{t} & \in C\left(I ; L_{\mu}^{2}\left(\mathbb{R}^{+} ; V_{2 \theta}\right)\right) ; \\
\eta_{t}^{t}+\eta_{s}^{t} & \in L^{\infty}\left(I ; L_{\mu}^{2}\left(\mathbb{R}^{+} ; V_{\theta}\right)\right) \cap L^{2}\left(I ; L_{\mu}^{2}\left(\mathbb{R}^{+} ; V_{2 \theta}\right)\right) .
\end{aligned}
$$

Hereafter, similar to Theorem 5, we can obtain the following.

Theorem 13 (existence and uniqueness of strong solutions). Assume that $f$ satisfy (2) and (4), $g \in L^{2}(\Omega)$, and (7)-(9) hold. Then, for any given $z_{0}=\left(u_{0}, u_{t 0}, \eta^{0}\right) \in \mathscr{H}_{2}$, and any $T>$ 0 , there exists a unique solution $z(t)=\left(u, u_{t}, \eta^{t}\right) \in \mathscr{H}_{2}$ for problem (17)-(18), in the sense that

$$
z \in L^{\infty}\left([0, T] ; \mathscr{H}_{2}\right) \cap C\left([0, T] ; \mathscr{H}_{2}\right) .
$$

By Theorem 13, we can define the strong solution operator as follows:

$$
\begin{aligned}
S(t): \mathscr{H}_{2} & \longrightarrow \mathscr{H}_{2}, \\
z_{0} & =\left(u_{0}, u_{t 0}, \eta^{0}\right) \longrightarrow \\
\left(u(t), u_{t}(t), \eta^{t}\right) & =S(t) z_{0} .
\end{aligned}
$$

Evidently, $\{S(t)\}_{t \geqslant 0}$ is a solution semigroup.
4.2. The Existence of Bounded Absorbing Set. At first, we will prove the existence of bounded absorbing set in $\mathscr{H}_{2}$.

Theorem 14 (bounded absorbing in $\mathscr{H}_{2}$ ). Let the nonlinearity $f$ satisfy (2), (5), and (6), $g \in L^{2}(\Omega)$, (7)-(9) hold, and $B_{1}$ be bounded subset of $\mathscr{H}_{2}$. If $z(t)$ is a solution of (14) and (17) with initial data $z_{0} \in B_{1}$, then there is a positive constant $\mu_{1}$ such that, for any bounded subset $B \subset \mathscr{H}_{2}$, there exists $t_{1}=$ $t_{1}\left(\|B\|_{\mathscr{H}_{2}}\right)$ such that

$$
\left\|S(t) z_{0}\right\|_{\mathscr{C}_{2}} \leqslant \mu_{1}, \quad \forall t \geqslant t_{1}, \quad z_{0} \in B .
$$

Proof. Taking the $L^{2}$ inner product of (17) with $A^{\theta} u_{t}+\rho A^{\theta} u$, we get that

$$
\begin{aligned}
\frac{1}{2} \frac{d}{d t} & \left(\|u\|_{V_{2 \theta}}^{2}+\left\|u_{t}+\rho u\right\|_{V_{\theta}}^{2}+\left\|\eta^{t}\right\|_{\mu, V_{2 \theta}}^{2}\right)+\rho\|u\|_{V_{2 \theta}}^{2} \\
& +(\alpha-\rho)\left\|u_{t}+\rho u\right\|_{V_{\theta}}^{2} \\
& -\rho(\alpha-\rho)\left\langle u, u_{t}+\rho u\right\rangle_{V_{\theta}}+\left\langle\eta^{t}, \eta_{s}^{t}\right\rangle_{\mu, V_{2 \theta}} \\
& +\rho\left\langle\eta^{t}, u\right\rangle_{\mu, V_{2 \theta}}+\left\langle f(u), A^{\theta}\left(u_{t}+\rho u\right)\right\rangle \\
= & \left\langle g, A^{\theta}\left(u_{t}+\rho u\right)\right\rangle .
\end{aligned}
$$

Similar to (35), we have

$$
\begin{aligned}
& \frac{1}{2} \frac{d}{d t}\left(\|u\|_{V_{2 \theta}}^{2}+\left\|u_{t}+\rho u\right\|_{V_{\theta}}^{2}+\left\|\eta^{t}\right\|_{\mu, V_{2 \theta}}^{2}\right) \\
& \quad+\rho\left(1-\frac{\alpha \rho}{\lambda_{1}^{\theta}}-\frac{k_{0} \rho}{\delta}\right)\|u\|_{V_{2 \theta}}^{2} \\
& \quad+\left(\frac{1}{2} \alpha-\rho\right)\left\|u_{t}+\rho u\right\|_{V_{\theta}}^{2}+\frac{\delta}{4}\left\|\eta^{t}\right\|_{\mu, V_{2 \theta}}^{2} \\
& \quad+\left\langle f(u), A^{\theta}\left(u_{t}+\rho u\right)\right\rangle \leqslant\left\langle g, A^{\theta}\left(u_{t}+\rho u\right)\right\rangle .
\end{aligned}
$$

About the nonlinearity, from (5), (6), and (44), these yield

$$
\begin{aligned}
- & \left\langle f(u), A^{\theta} u_{t}+\rho A^{\theta} u\right\rangle \\
& =-\left\langle f^{\prime}(\tau) u, A^{\theta}\left(u_{t}+\rho u\right)\right\rangle \\
& \leqslant l\left|\left\langle u, A^{\theta}\left(u_{t}+\rho u\right)\right\rangle\right| \\
& =l\left|\left\langle A^{\theta / 2} u, A^{\theta / 2}\left(u_{t}+\rho u\right)\right\rangle\right| \\
& \leqslant \frac{\rho}{2}\left\|u_{t}+\rho u\right\|_{V_{\theta}}^{2}+\frac{l^{2}}{2 \rho}\|u\|_{V_{\theta}}^{2} \\
& \leqslant \frac{\rho}{2}\left\|u_{t}+\rho u\right\|_{V_{\theta}}^{2}+C,
\end{aligned}
$$

where $0 \leqslant \tau \leqslant u($ or $u \leqslant \tau \leqslant 0)$ and $0 \leqslant f^{\prime}(\tau) \leqslant l$.

For the forcing term, we see easily

$$
-\left\langle g, A^{\theta}\left(u_{t}+\rho u\right)\right\rangle=-\frac{d}{d t}\left\langle g, A^{\theta} u\right\rangle-\rho\left\langle g, A^{\theta} u\right\rangle,
$$


and since $g \in L^{2}(\Omega)$, then

$$
\left\langle g, A^{\theta} u\right\rangle \leqslant\|g\|^{2}+\frac{1}{4}\|u\|_{V_{2 \theta}}^{2} .
$$

Taking $\rho$ small enough, we then obtain that

$$
\begin{aligned}
1-\frac{\alpha \rho}{\lambda_{1}^{\theta}}-\frac{k_{0} \rho}{\delta} & \geqslant \frac{1}{2}, \\
\frac{1}{2} \alpha-\frac{3 \rho}{2} & \geqslant \frac{\alpha}{4} .
\end{aligned}
$$

In combination with the above estimates, we can deduce that, for all $t \geqslant t_{0}$,

$$
\begin{aligned}
& \frac{1}{2} \frac{d}{d t}\left(\|u\|_{V_{2 \theta}}^{2}+\left\|u_{t}+\rho u\right\|_{V_{\theta}}^{2}+\left\|\eta^{t}\right\|_{\mu, V_{2 \theta}}^{2}-2\left\langle g, A^{\theta} u\right\rangle\right. \\
& +\bar{C})+\frac{\beta_{3}}{2}\left(\|u\|_{V_{2 \theta}}^{2}+\left\|u_{t}+\rho u\right\|_{V_{\theta}}^{2}+\left\|\eta^{t}\right\|_{\mu, V_{2 \theta}}^{2}\right. \\
& \left.-2\left\langle g, A^{\theta} u\right\rangle+\bar{C}\right) \leqslant C,
\end{aligned}
$$

where $\bar{C}=2\|g\|^{2}$ and $\beta_{3}=\min \{\rho, \alpha / 2, \delta / 2\}$.

From (102), there exist constants $\bar{C}_{1}, \bar{C}_{2}$, such that

$$
\begin{gathered}
\bar{C}_{1}\|z(t)\|_{\mathscr{H}_{2}}^{2} \leqslant \frac{1}{2}\left(\|u\|_{V_{2 \theta}}^{2}+\left\|u_{t}+\rho u\right\|_{V_{\theta}}^{2}+\left\|\eta^{t}\right\|_{\mu, V_{2 \theta}}^{2}\right. \\
\left.-2\left\langle g, A^{\theta} u\right\rangle+\bar{C}\right) \leqslant \bar{C}_{2}\|z(t)\|_{\mathscr{H}_{2}}^{2} ;
\end{gathered}
$$

therefore, applying Gronwall lemma, we have

$$
\|z(t)\|_{\mathscr{H}_{2}}^{2} \leqslant \frac{\bar{C}_{2}}{\bar{C}_{1}}\|z(0)\|_{\mathscr{H}_{2}}^{2} e^{-\beta_{3} t}+\frac{C}{\beta_{3} \bar{C}_{1}} .
$$

Presuming that $\|z(0)\|_{\mathscr{C}_{2}}^{2} \leqslant R$, as $t \geqslant t_{1}=t_{1}\left(\left\|B_{1}\right\|_{\mathscr{H}_{2}}\right) \geqslant t_{0}$, we get that

$$
\|z(t)\|_{\mathscr{H}_{2}} \leqslant \mu_{1} .
$$

The proof is complete.

4.3. The Existence of Global Attractor in $\mathscr{H}_{2}$. According to the general theorem about the existence of global attractors of infinite-dimensional dynamical systems (see $[6,15])$, we also need to test the asymptotic compactness of $\{S(t)\}_{t \geqslant 0}$ in $\mathscr{H}_{2}$.

Similarly, we also gain the corresponding existence and uniqueness of solutions for (55) and (56); moreover, we can deduce that the solutions of (55) also form a semigroup. For the sake of convenience, we denote the solution operators of (55) and (56) by $\left\{S_{1}(t)\right\}_{t \geqslant 0}$ and $\left\{S_{2}(t, \cdot)\right\}_{t \geqslant 0}$, respectively. Then, for every $z_{0} \in \mathscr{H}_{2}$, we have

$$
z(t)=S(t) z_{0}=S_{1}(t) z_{0}+S_{2}\left(t, z_{0}\right), \quad \forall t \geqslant 0 .
$$

After that, the necessary condition of asymptotic smoothness will be verified.

At first, akin to the proof of Lemma 7, the following results about the solution $z_{1}(t)$ of (55) in $\mathscr{H}_{2}$ can be obtained.

Lemma 15. Let $f_{0}$ satisfy (48)-(50), $g \in L^{2}(\Omega)$, and (7)-(9) hold. Then, for any $\varepsilon_{1}>0$, there is a constant $\epsilon=\epsilon\left(\varepsilon_{1}, g\right)$ and $t_{1} \geqslant t_{0}^{*}>t_{0}$, such that the solutions of (55) satisfy the following estimates:

$$
\begin{aligned}
\left\|S_{1}(t) z_{0}\right\|_{\mathscr{H}_{2}}^{2} & =\frac{1}{2}\left(\left\|v^{\epsilon}\right\|_{V_{2 \theta}}^{2}+\left\|v_{t}^{\epsilon}\right\|_{V_{\theta}}^{2}+\left\|\zeta^{t \epsilon}\right\|_{\mu, V_{2 \theta}}^{2}\right) \\
& \leqslant Q\left(\left\|z_{0}\right\|_{\mathscr{H}_{2}}\right) e^{-\beta_{4} t}+\varepsilon_{1}, \quad \forall t \geqslant t_{1} ;
\end{aligned}
$$

here $Q(\cdot)$ is an increasing function on $[0, \infty)$ and $\beta_{4}$ only depends on $\alpha, \rho$, and $\delta$.

Proof. Multiplying (55) by $A^{\theta} v_{t}^{\epsilon}+\rho A^{\theta} v^{\epsilon}$, we get that

$$
\begin{aligned}
\frac{1}{2} \frac{d}{d t} & \left(\left\|v^{\epsilon}\right\|_{V_{2 \theta}}^{2}+\left\|v_{t}^{\epsilon}+\rho v^{\epsilon}\right\|_{V_{\theta}}^{2}+\left\|\zeta^{\epsilon}\right\|_{\mu, V_{2 \theta}}^{2}\right)+\rho\left\|v^{\epsilon}\right\|_{V_{2 \theta}}^{2} \\
& +(\alpha-\rho)\left\|v_{t}^{\epsilon}+\rho v^{\epsilon}\right\|_{V_{\theta}}^{2} \\
& -\rho(\alpha-\rho)\left\langle v^{\epsilon}, v_{t}^{\epsilon}+\rho v^{\epsilon}\right\rangle_{V_{\theta}}+\left\langle\zeta^{t \epsilon}, \zeta_{s}^{t \epsilon}\right\rangle_{\mu, V_{2 \theta}} \\
& +\rho\left\langle\zeta^{t \epsilon}, v^{\epsilon}\right\rangle_{\mu, V_{2 \theta}} \\
= & -\left\langle f_{0}\left(v^{\epsilon}\right), A^{\theta}\left(v_{t}^{\epsilon}+\rho v^{\epsilon}\right)\right\rangle \\
& +\left\langle g-g^{\epsilon}, A^{\theta}\left(v_{t}^{\epsilon}+\rho v^{\epsilon}\right)\right\rangle .
\end{aligned}
$$

For the forcing term, we have

$$
\begin{aligned}
& -\left\langle g-g^{\epsilon}, A^{\theta}\left(v_{t}^{\epsilon}+\rho v^{\epsilon}\right)\right\rangle \\
& =-\frac{d}{d t}\left\langle g-g^{\epsilon}, A^{\theta} v^{\epsilon}\right\rangle-\rho\left\langle g-g^{\epsilon}, A^{\theta} v^{\epsilon}\right\rangle, \\
& \left\langle g-g^{\epsilon}, A^{\theta} v^{\epsilon}\right\rangle \leqslant\left\|g-g^{\epsilon}\right\|^{2}+\frac{1}{4}\left\|v^{\epsilon}\right\|_{V_{2 \theta}}^{2} .
\end{aligned}
$$

About the nonlinearity, similar to (100) and from (48), (50), and (70), as $t \geqslant t_{0}^{*}>t_{0}$, we see

$$
\begin{aligned}
& -\left\langle f_{0}\left(v^{\epsilon}\right), A^{\theta} v_{t}^{\epsilon}+\rho A^{\theta} v^{\epsilon}\right\rangle \\
& \leqslant \frac{\alpha}{8}\left\|v_{t}^{\epsilon}+\rho v^{\epsilon}\right\|_{V_{\theta}}^{2}+\frac{2 l_{1}^{2}}{\alpha}\left\|v^{\epsilon}\right\|_{V_{\theta}}^{2} \\
& \leqslant \frac{\alpha}{8}\left\|v_{t}^{\epsilon}+\rho v^{\epsilon}\right\|_{V_{\theta}}^{2}+C \epsilon .
\end{aligned}
$$

Similarly,

$$
\begin{aligned}
\frac{1}{2} & \frac{d}{d t}\left(\left\|v^{\epsilon}\right\|_{V_{2 \theta}}^{2}+\left\|v_{t}^{\epsilon}+\rho v^{\epsilon}\right\|_{V_{\theta}}^{2}+\left\|\zeta^{\epsilon \epsilon}\right\|_{\mu, V_{2 \theta}}^{2}\right. \\
& \left.-2\left\langle g-g^{\epsilon}, A^{\theta} v^{\epsilon}\right\rangle+\check{C}\right)+\left(\frac{\rho}{2}-\frac{k_{0} \rho^{2}}{\delta}\right)\left\|v^{\epsilon}\right\|_{V_{2 \theta}}^{2} \\
& +\frac{\alpha}{8}\left\|v_{t}^{\epsilon}+\rho v^{\epsilon}\right\|_{V_{\theta}}^{2}+\frac{\delta}{4}\left\|\zeta^{t \epsilon}\right\|_{\mu, V_{2 \theta}}^{2}-\rho\langle g \\
& \left.-g^{\epsilon}, A^{\theta} v^{\epsilon}\right\rangle+\rho \check{C} \leqslant \rho \check{C}+C \epsilon<C \epsilon ;
\end{aligned}
$$

here, $\check{C}=2\left\|g-g^{\epsilon}\right\|^{2}<2 \epsilon^{2}$. 
Then, substituting the above estimates into (110), this yields

$$
\begin{aligned}
& \frac{1}{2} \frac{d}{d t}\left(\left\|v^{\epsilon}\right\|_{V_{2 \theta}}^{2}+\left\|v_{t}^{\epsilon}+\rho v^{\epsilon}\right\|_{V_{\theta}}^{2}+\left\|\zeta^{\epsilon \epsilon}\right\|_{\mu, V_{2 \theta}}^{2}\right. \\
& \left.-2\left\langle g-g^{\epsilon}, A^{\theta} v^{\epsilon}\right\rangle+\check{C}\right)+\frac{\beta_{4}}{2}\left(\left\|v^{\epsilon}\right\|_{V_{2 \theta}}^{2}\right. \\
& +\left\|v_{t}^{\epsilon}+\rho v^{\epsilon}\right\|_{V_{\theta}}^{2}+\left\|\zeta^{\epsilon}\right\|_{\mu, V_{2 \theta}}^{2}-2\left\langle g-g^{\epsilon}, A^{\theta} v^{\epsilon}\right\rangle \\
& +\check{C}) \leqslant C \epsilon,
\end{aligned}
$$

where $\rho / 2-k_{0} \rho^{2} / \delta \geqslant \rho / 4, \beta_{4}=\min \{\rho / 2, \alpha / 4, \delta / 2\}$.

Obviously, there exist constants $\check{C}_{1}, \check{C}_{2}$, such that

$$
\begin{gathered}
\check{C}_{1}\left\|z_{1}(t)\right\|_{\mathscr{C}_{2}}^{2} \leqslant \frac{1}{2}\left(\left\|v^{\epsilon}\right\|_{V_{2 \theta}}^{2}+\left\|v_{t}^{\epsilon}+\rho v^{\epsilon}\right\|_{V_{\theta}}^{2}+\left\|\zeta^{\epsilon \epsilon}\right\|_{\mu, V_{2 \theta}}^{2}\right. \\
\left.-2\left\langle g-g^{\epsilon}, A^{\theta} v^{\epsilon}\right\rangle+\check{C}\right) \leqslant \check{C}_{2}\left\|z_{1}(t)\right\|_{\mathscr{H}_{2}}^{2} .
\end{gathered}
$$

From Gronwall lemma, for all $t \geqslant t_{1} \geqslant t_{0}^{*}$, we obtain

$$
\begin{aligned}
\left\|z_{1}(t)\right\|_{\mathscr{H}_{2}}^{2} & \leqslant \frac{\check{C}_{2}}{\check{C}_{1}}\|z(0)\|_{\mathscr{H}_{2}}^{2} e^{-\beta_{4} t}+\frac{C \epsilon}{\beta_{4} \check{C}_{1}} \\
& \leqslant Q\left(\left\|z_{0}\right\|_{\mathscr{H}_{2}}\right) e^{-\beta_{4} t}+\varepsilon_{1} .
\end{aligned}
$$

The proof is complete.

Lemma 16. Let the nonlinearity $f$ satisfy (2)-(6) and (51)(52), $g \in L^{2}(\Omega)$, and (7)-(9) hold. Then, for any given $T>0$ and $\epsilon>0$, there exists a position constant

$$
N_{1}=N_{1}\left(T,\|g\|,\left\|z_{0}\right\|_{\mathscr{H}_{2}}, \delta\right),
$$

such that the solutions of (56) satisfy

$$
\begin{aligned}
& \left\|S_{2}\left(T, z_{0}\right)\right\|_{\mathscr{H}_{2+\sigma}}^{2} \\
& \quad=\frac{1}{2}\left(\left\|w^{\epsilon}\right\|_{V_{2 \theta+\sigma}}^{2}+\left\|w_{t}^{\epsilon}\right\|_{V_{\theta+\sigma}}^{2}+\left\|\xi^{T e}\right\|_{\mu, V_{2 \theta+\sigma}}^{2}\right) \leqslant N_{1},
\end{aligned}
$$

where $\sigma=\min \{1,((n+2) \theta-2 n) /(n-2), 2 \theta / 3, \theta-(n-2 \theta) \gamma / 2\}$.

Proof. Multiplying (56) by $A^{\theta+\sigma}\left(w_{t}^{\epsilon}+\rho w^{\epsilon}\right)$, we obtain that

$$
\begin{aligned}
\frac{1}{2} \frac{d}{d t} & \left(\left\|w^{\epsilon}\right\|_{V_{2 \theta+\sigma}}^{2}+\left\|w_{t}^{\epsilon}+\rho w^{\epsilon}\right\|_{V_{\theta+\sigma}}^{2}+\left\|\xi^{t \epsilon}\right\|_{\mu, V_{2 \theta+\sigma}}^{2}\right) \\
& +\rho\left\|w^{\epsilon}\right\|_{V_{2 \theta+\sigma}}^{2}+(\alpha-\rho)\left\|w_{t}^{\epsilon}+\rho w\right\|_{V_{\theta+\sigma}}^{2} \\
& -\rho(\alpha-\rho)\left\langle w^{\epsilon}, w_{t}^{\epsilon}+\rho w^{\epsilon}\right\rangle_{V_{\theta+\sigma}} \\
& +\left\langle\xi^{t \epsilon}, \xi_{s}^{t \epsilon}\right\rangle_{\mu, V_{2 \theta+\sigma}}+\rho\left\langle\xi^{t \epsilon}, w^{\epsilon}\right\rangle_{\mu, V_{2 \theta+\sigma}} \\
= & -\left\langle f(u)-f_{0}\left(v^{\epsilon}\right), A^{\theta+\sigma}\left(w_{t}^{\epsilon}+\rho w^{\epsilon}\right)\right\rangle \\
& +\left\langle g^{\epsilon}, A^{\theta+\sigma}\left(w_{t}^{\epsilon}+\rho w^{\epsilon}\right)\right\rangle .
\end{aligned}
$$

Similarly, choosing $\rho$ to be sufficiently small, we can obtain that

$$
\begin{aligned}
\frac{1}{2} \frac{d}{d t} & \left(\left\|w^{\epsilon}\right\|_{V_{2 \theta+\sigma}}^{2}+\left\|w_{t}^{\epsilon}+\rho w^{\epsilon}\right\|_{V_{\theta+\sigma}}^{2}+\left\|\xi^{\epsilon \epsilon}\right\|_{\mu, V_{2 \theta+\sigma}}^{2}\right) \\
& +\frac{\rho}{2}\left\|w^{\epsilon}\right\|_{V_{2 \theta+\sigma}}^{2}+\frac{\alpha}{4}\left\|w_{t}^{\epsilon}+\rho w\right\|_{V_{\theta+\sigma}}^{2} \\
& +\frac{\delta}{4}\left\|\xi^{t \epsilon}\right\|_{\mu, V_{2 \theta+\sigma}}^{2} \\
= & -\left\langle f(u)-f_{0}\left(v^{\epsilon}\right), A^{\theta+\sigma}\left(w_{t}^{\epsilon}+\rho w^{\epsilon}\right)\right\rangle \\
& +\left\langle g^{\epsilon}, A^{\theta+\sigma}\left(w_{t}^{\epsilon}+\rho w^{\epsilon}\right)\right\rangle .
\end{aligned}
$$

Clearly

$$
\begin{aligned}
\left\langle f(u)-f_{0}\left(v^{\epsilon}\right), A^{\theta+\sigma}\left(w_{t}^{\epsilon}+\rho w^{\epsilon}\right)\right\rangle \\
=\left\langle f(u)-f\left(v^{\epsilon}\right), A^{\theta+\sigma}\left(w_{t}^{\epsilon}+\rho w^{\epsilon}\right)\right\rangle \\
+\left\langle f_{1}\left(v^{\epsilon}\right), A^{\theta+\sigma}\left(w_{t}^{\epsilon}+\rho w^{\epsilon}\right)\right\rangle .
\end{aligned}
$$

We deal with the terms in (121) one by one.

First, from (4), we obtain

$$
\begin{aligned}
& -\left\langle f(u)-f\left(v^{\epsilon}\right), A^{\theta+\sigma}\left(w_{t}^{\epsilon}+\rho w^{\epsilon}\right)\right\rangle \\
& \leqslant \mid \int_{\Omega}\left(f^{\prime}(u) A^{3 \sigma / 4+\theta / 2} u-f^{\prime}\left(v^{\epsilon}\right) A^{3 \sigma / 4+\theta / 2} v^{\epsilon}\right) \\
& \cdot A^{\sigma / 4+\theta / 2}\left(w_{t}^{\epsilon}+\rho w^{\epsilon}\right) d x \mid \\
& \leqslant C \int_{\Omega}\left(1+|u|^{4 /(n-4)}+\left|v^{\epsilon}\right|^{4 /(n-4)}\right)\left|A^{3 \sigma / 4+\theta / 2} w^{\epsilon}\right| \\
& \cdot\left|A^{\sigma / 4+\theta / 2}\left(w_{t}^{\epsilon}+\rho w^{\epsilon}\right)\right| d x \\
& +2 C \int_{\Omega}\left(1+|u|^{4 /(n-4)}+\left|v^{\epsilon}\right|^{4 /(n-4)}\right)\left|A^{3 \sigma / 4+\theta / 2} v^{\epsilon}\right| \\
& \cdot\left|A^{\sigma / 4+\theta / 2}\left(w_{t}^{\epsilon}+\rho w^{\epsilon}\right)\right| d x .
\end{aligned}
$$

Second, for $\theta \in[2 n /(n+2), n / 2)$, we have

$$
\begin{aligned}
& C \int_{\Omega}\left(1+|u|^{4 /(n-4)}+\left|v^{\epsilon}\right|^{4 /(n-4)}\right)\left|A^{3 \sigma / 4+\theta / 2} w^{\epsilon}\right| \\
& \cdot\left|A^{\sigma / 4+\theta / 2}\left(w_{t}^{\epsilon}+\rho w^{\epsilon}\right)\right| d x \\
& \quad \leqslant C\left(\int_{\Omega}\left(1+|u|^{4 /(n-4)}+\left|v^{\epsilon}\right|^{4 /(n-4)}\right)^{n / \theta} d x\right)^{\theta / n} \\
& \cdot\left(\int_{\Omega}\left|A^{3 \sigma / 4+\theta / 2} w^{\epsilon}\right|^{2 n /(n-2(\theta-\sigma / 2))} d x\right)^{(n-2(\theta-\sigma / 2)) / 2 n} \\
& \cdot\left(\int_{\Omega}\left|A^{\sigma / 4+\theta / 2}\left(w_{t}^{\epsilon}+\rho w^{\epsilon}\right)\right|^{2 n /(n-\sigma)} d x\right)^{(n-\sigma) / 2 n}
\end{aligned}
$$




$$
\begin{aligned}
& \leqslant C\left(1+\|u\|_{L^{4 n /(n-4) \theta}}^{4 /(n-4)}+\left\|v^{\epsilon}\right\|_{L^{4 n /(n-4) \theta}}^{4 /(n-4)}\right) \\
& \cdot\left\|A^{3 \sigma / 4+\theta / 2} w^{\epsilon}\right\|_{L^{2 n /(n-2(\theta-\sigma / 2))}} \\
& \cdot\left\|A^{\sigma / 4+\theta / 2}\left(w_{t}^{\epsilon}+\rho w^{\epsilon}\right)\right\|_{L^{2 n /(n-\sigma)}} \\
& \leqslant C\left(1+\|u\|_{L^{2 n /(n-4 \theta)}}^{4 /(n-4)}+\left\|v^{\epsilon}\right\|_{L^{2 n /(n-4 \theta)}}^{4 /(n-4)}\right) \\
& \cdot\left\|A^{3 \sigma / 4+\theta / 2} w^{\epsilon}\right\|_{L^{2 n /(n-2(\theta-\sigma / 2))}} \\
& \cdot\left\|A^{\sigma / 4+\theta / 2}\left(w_{t}^{\epsilon}+\rho w^{\epsilon}\right)\right\|_{L^{2 n /(n-\sigma)}} \\
& \leqslant C\left(1+\left\|A^{\theta} u\right\|^{4 /(n-4)}+\left\|A^{\theta} v^{\epsilon}\right\|^{4 /(n-4)}\right) \\
& \cdot\left\|A^{(2 \theta+\sigma) / 2} w^{\epsilon}\right\| \cdot\left\|A^{(\theta+\sigma) / 2}\left(w_{t}^{\epsilon}+\rho w^{\epsilon}\right)\right\| \\
& \leqslant C{ }_{\mu_{1}, \varepsilon_{1}}\left\|A^{(2 \theta+\sigma) / 2} w^{\epsilon}\right\|^{2} \\
& +\frac{\alpha}{4}\left\|A^{(\theta+\sigma) / 2}\left(w_{t}^{\epsilon}+\rho w^{\epsilon}\right)\right\|^{2},
\end{aligned}
$$

where $4 n /(n-4) \theta \leqslant 2 n /(n-4 \theta)$, and we have used the embedding $V_{2 \theta}=D\left(A^{\theta}\right) \hookrightarrow L^{2 n /(n-4 \theta)} \hookrightarrow L^{4 n /(n-4) \theta}, V_{\theta-\sigma / 2}=$ $D\left(A^{(2 \theta-\sigma) / 4}\right) \hookrightarrow L^{2 n /(n-2(\theta-\sigma / 2))}, V_{\sigma / 2}=D\left(A^{\sigma / 4}\right) \hookrightarrow L^{2 n /(n-\sigma)}$.

Third, from (53), we obtain that

$$
\begin{aligned}
& 2 C \int_{\Omega}\left(1+|u|^{4 /(n-4)}+\left|v^{\epsilon}\right|^{4 /(n-4)}\right)\left|A^{3 \sigma / 4+\theta / 2} v^{\epsilon}\right| \\
& \cdot\left|A^{\sigma / 4+\theta / 2}\left(w_{t}^{\epsilon}+\rho w^{\epsilon}\right)\right| d x \leqslant 2 C\left(\int_{\Omega}(1\right. \\
& \left.\left.+|u|^{4 /(n-4)}+\left|v^{\epsilon}\right|^{4 /(n-4)}\right)^{n /(\theta-\sigma)} d x\right)^{(\theta-\sigma) / n} \\
& \cdot\left(\int_{\Omega}\left|A^{3 \sigma / 4+\theta / 2} v^{\epsilon}\right|^{2 n /(n-2(\theta-3 \sigma / 2))} d x\right)^{(n-2(\theta-3 \sigma / 2)) / 2 n} \\
& \cdot\left(\int_{\Omega}\left|A^{\sigma / 4+\theta / 2}\left(w_{t}^{\epsilon}+\rho w^{\epsilon}\right)\right|^{2 n /(n-\sigma)} d x\right)^{(n-\sigma) / 2 n} \\
& \quad \leqslant 2 C\left(1+\|u\|_{L^{4 n /(n-4)(\theta-\sigma)}}^{4 /(n-4)}+\left\|v^{\epsilon}\right\|_{L^{4 n /(n-4)(\theta-\sigma)}}^{4 /(n-4)}\right) \\
& \cdot\left\|A^{3 \sigma / 4+\theta / 2} v^{\epsilon}\right\|_{L^{2 n /(n-2(\theta-3 \sigma / 2))}} \| A^{\sigma / 4+\theta / 2}\left(w_{t}^{\epsilon}\right. \\
& \left.+\rho w^{\epsilon}\right) \|_{L^{2 n /(n-\sigma)}} \leqslant 2 C\left(1+\|u\|_{L^{2 n /(n-4 \theta)}}^{4 /(n-4)}+\left\|v^{\epsilon}\right\|_{L^{2 n /(n-4 \theta)}}^{4 /(n-4)}\right) \\
& \quad \leqslant C_{\mu_{1}, \varepsilon_{1}}+\frac{\alpha}{4}\left\|A^{(\theta+\sigma) / 2}\left(w_{t}^{\epsilon}+\rho w^{\epsilon}\right)\right\|^{2} \\
& +\left\|A^{3 \sigma / 4+\theta / 2} v^{\epsilon}\right\|_{L^{2 n /(n-2(\theta-3 \sigma / 2))}} \| A^{\sigma / 4+\theta / 2}\left(w_{t}^{\epsilon}\right. \\
& \left.+\rho w^{\epsilon}\right)\left\|_{L^{2 n /(n-\sigma)}} \leqslant 2 C\left(1+\left\|A^{\theta} u\right\|^{\epsilon} \|^{4 /(n-4)}\right)\right\| A^{\theta} v^{\epsilon}\|\cdot\| A^{(\theta+\sigma-\sigma) / 2}\left(w_{t}^{\epsilon}+\rho w^{\epsilon}\right) \|
\end{aligned}
$$

where $4 n /(n-4)(\theta-\sigma) \leqslant 2 n /(n-4 \theta)$, and we have used the embedding $V_{2 \theta}=D\left(A^{\theta}\right) \hookrightarrow L^{2 n /(n-4 \theta)} \hookrightarrow$ $L^{4 n /(n-4)(\theta-\sigma)}, V_{\theta-3 \sigma / 2}=D\left(A^{(2 \theta-3 \sigma) / 4}\right) \hookrightarrow L^{2 n /(n-2(\theta-3 \sigma / 2))}$, $V_{\sigma / 2}=D\left(A^{\sigma / 4}\right) \hookrightarrow L^{2 n /(n-\sigma)}$.

Finally, by virtue of (51), we get

$$
\begin{aligned}
& -\left\langle f_{1}\left(v^{\epsilon}\right), A^{\theta+\sigma}\left(w_{t}^{\epsilon}+\rho w^{\epsilon}\right)\right\rangle=-\int_{\Omega} f_{1}^{\prime}\left(v^{\epsilon}\right) \\
& \cdot A^{3 \sigma / 4+\theta / 2} v^{\epsilon} \cdot A^{\sigma / 4+\theta / 2}\left(w_{t}^{\epsilon}+\rho w^{\epsilon}\right) d x \\
& \leqslant C \int_{\Omega}\left(1+\left|v^{\epsilon}\right|^{\gamma}\right)\left|A^{3 \sigma / 4+\theta / 2} v^{\epsilon}\right| \\
& \cdot\left|A^{\sigma / 4+\theta / 2}\left(w_{t}^{\epsilon}+\rho w^{\epsilon}\right)\right| d x \\
& \leqslant C\left(\int_{\Omega}\left(1+\left|v^{\epsilon}\right|^{\gamma}\right)^{n /(\theta-\sigma)} d x\right)^{(\theta-\sigma) / n} \\
& \cdot\left(\int_{\Omega}\left|A^{3 \sigma / 4+\theta / 2} v^{\epsilon}\right|^{2 n /(n-2(\theta-3 \sigma / 2))} d x\right)^{(n-2(\theta-3 \sigma / 2)) / 2 n} \\
& \cdot\left(\int_{\Omega}\left|A^{\sigma / 4+\theta / 2}\left(w_{t}^{\epsilon}+\rho w^{\epsilon}\right)\right|^{2 n /(n-\sigma)} d x\right)^{(n-\sigma) / 2 n} \\
& \leqslant C\left(1+\left\|v^{\epsilon}\right\|_{L^{n \gamma /(\theta-\sigma)}}^{\gamma}\right)\left\|A^{3 \sigma / 4+\theta / 2} v^{\epsilon}\right\|_{L^{2 n /(n-2(\theta-3 \sigma / 2))}} \\
& \cdot\left\|A^{\sigma / 4+\theta / 2}\left(w_{t}^{\epsilon}+\rho w^{\epsilon}\right)\right\|_{L^{2 n /(n-\sigma)}} \leqslant C(1 \\
& \left.+\left\|v^{\epsilon}\right\|_{L^{2 n /(n-4 \theta)}}^{\gamma}\right)\left\|A^{3 \sigma / 4+\theta / 2} v^{\epsilon}\right\|_{L^{2 n /(n-2(\theta-3 \sigma / 2))}} \\
& \cdot\left\|A^{\sigma / 4+\theta / 2}\left(w_{t}^{\epsilon}+\rho w^{\epsilon}\right)\right\|_{L^{2 n /(n-\sigma)}} \leqslant C\left(1+\left\|A^{\theta} v^{\epsilon}\right\|^{\gamma}\right) \\
& \cdot\left\|A^{\theta} v^{\epsilon}\right\|\left\|A^{(\theta+\sigma) / 2}\left(w_{t}^{\epsilon}+\rho w^{\epsilon}\right)\right\| \leqslant C_{\varepsilon_{1}} \\
& +\frac{\alpha}{4}\left\|A^{(\theta+\sigma) / 2}\left(w_{t}^{\epsilon}+\rho w^{\epsilon}\right)\right\|^{2},
\end{aligned}
$$

where we have employed the inequality $\sigma \leqslant \theta-(n-$ $4 \theta) \gamma / 2, n \gamma /(\theta-\sigma) \leqslant 2 n /(n-4 \theta)$ and used the embedding $V_{2 \theta} \hookrightarrow L^{2 n /(n-4 \theta)} \hookrightarrow L^{n \gamma /(\theta-\sigma)}, V_{\sigma / 2}=D\left(A^{\sigma / 4}\right) \hookrightarrow L^{2 n /(n-\sigma)}$, and $V_{\theta-3 \sigma / 2}=D\left(A^{(2 \theta-3 \sigma) / 4}\right) \hookrightarrow L^{2 n /(n-2(\theta-3 \sigma / 2))}$.

At the same time, due to $0<\sigma<1$, we have

$$
\begin{aligned}
& \left\langle g^{\epsilon}, A^{\theta+\sigma}\left(w_{t}^{\epsilon}+\rho w^{\epsilon}\right)\right\rangle \\
& =\frac{d}{d t}\left\langle g^{\epsilon}, A^{\theta+\sigma} w^{\epsilon}\right\rangle+\rho\left\langle g^{\epsilon}, A^{\theta+\sigma} w^{\epsilon}\right\rangle, \\
& \left\langle g^{\epsilon}, A^{\theta+\sigma} w^{\epsilon}\right\rangle \leqslant\left\|g^{\epsilon}\right\|_{V_{1}}\left\|A^{\theta+\sigma-1 / 2} w^{\epsilon}\right\| \\
& \leqslant \frac{1}{2 \lambda_{1}^{1-\sigma}}\left\|g^{\epsilon}\right\|_{V_{1}}^{2}+\frac{\lambda_{1}^{1-\sigma}}{2}\left\|A^{\theta+\sigma-1 / 2} w^{\epsilon}\right\|^{2} \\
& \leqslant \frac{1}{2 \lambda_{1}^{1-\sigma}}\left\|g^{\epsilon}\right\|_{V_{1}}^{2}+\frac{1}{2}\left\|A^{(2 \theta+\sigma) / 2} w^{\epsilon}\right\|^{2} .
\end{aligned}
$$


Combining the above estimates, we can deduce that

$$
\begin{aligned}
& \frac{1}{2} \frac{d}{d t}\left(\left\|w^{\epsilon}\right\|_{V_{2 \theta+\sigma}}^{2}+\left\|w_{t}^{\epsilon}+\rho w^{\epsilon}\right\|_{V_{\theta+\sigma}}^{2}+\left\|\xi^{t \epsilon}\right\|_{\mu, V_{2 \theta+\sigma}}^{2}\right. \\
& \left.\quad-2\left\langle g^{\epsilon}, A^{\theta+\sigma} w^{\epsilon}\right\rangle+\breve{C}\right) \leqslant \frac{\beta_{5}}{2}\left(\left\|w^{\epsilon}\right\|_{V_{2 \theta+\sigma}}^{2}\right. \\
& +\left\|w_{t}^{\epsilon}+\rho w^{\epsilon}\right\|_{V_{\theta+\sigma}}^{2}+\left\|\xi^{\epsilon \epsilon}\right\|_{\mu, V_{2 \theta+\sigma}}^{2}-2\left\langle g^{\epsilon}, A^{\theta+\sigma} w^{\epsilon}\right\rangle \\
& +\breve{C})+\mathrm{C}
\end{aligned}
$$

where $\breve{C}=\left(1 / \lambda_{1}^{1-\sigma}\right)\left\|g^{\epsilon}\right\|_{V_{1}}^{2}, \beta_{5}=\max \left\{2 C_{\mu_{1}, \varepsilon_{1}}-\rho, \alpha, \delta / 2\right\}$, and the constant $C$ only depends on $\left\|g^{\epsilon}\right\|,\left\|z_{0}\right\|_{\mathscr{H}_{2}}$.

Applying the Gronwall inequality and integrating over $[0, t]$, we deduce that

$$
\begin{aligned}
& \frac{1}{2}\left(\left\|w^{\epsilon}(t)\right\|_{V_{2 \theta+\sigma}}^{2}+\left\|w_{t}^{\epsilon}(t)+\rho w^{\epsilon}(t)\right\|_{V_{\theta+\sigma}}^{2}\right. \\
& \left.+\left\|\xi^{t \epsilon}(s)\right\|_{\mu, V_{2 \theta+\sigma}}^{2}-2\left\langle g^{\epsilon}(x), A^{\theta+\sigma} w^{\epsilon}(t)\right\rangle+\breve{C}\right) \\
& \leqslant \frac{1}{2}\left(\left\|w^{\epsilon}(0)\right\|_{V_{2 \theta+\sigma}}^{2}+\left\|w_{t}^{\epsilon}(0)+\rho w^{\epsilon}(0)\right\|_{V_{\theta+\sigma}}^{2}\right. \\
& \left.+\left\|\xi^{0 \epsilon}(s)\right\|_{\mu, V_{2 \theta+\sigma}}^{2}-2\left\langle g^{\epsilon}(x), A^{\theta+\sigma} w^{\epsilon}(0)\right\rangle+\breve{C}\right) \\
& \cdot e^{\beta_{5} t}+\frac{C}{\beta_{5}}\left(e^{\beta_{5} t}-1\right) \leqslant\left(\frac{\breve{C}}{2}+\frac{C}{\beta_{5}}\right) e^{\beta_{5} t} .
\end{aligned}
$$

So, there exist constants $\breve{C}_{1}, \breve{C}_{2}$, such that

$$
\begin{aligned}
\breve{C}_{1} & \left\|z_{2}(t)\right\|_{\mathscr{H}_{2+\sigma}}^{2} \leqslant \frac{1}{2}\left(\left\|w^{\epsilon}(t)\right\|_{V_{2 \theta+\sigma}}^{2}\right. \\
& +\left\|w_{t}^{\epsilon}(t)+\rho w^{\epsilon}(t)\right\|_{V_{\theta+\sigma}}^{2}+\left\|\xi^{t \epsilon}(s)\right\|_{\mu, V_{2 \theta+\sigma}}^{2} \\
& \left.-2\left\langle g^{\epsilon}(x), A^{\theta+\sigma} w^{\epsilon}(t)\right\rangle+\breve{C}\right) \leqslant \breve{C}_{2}\left\|z_{2}(t)\right\|_{\mathscr{H}_{2+\sigma}}^{2} ;
\end{aligned}
$$

then

$$
\left\|z_{2}(t)\right\|_{\mathscr{H}_{2+\sigma}}^{2} \leqslant \frac{1}{\breve{C}_{1}}\left(\frac{\breve{C}}{2}+\frac{C}{\beta_{5}}\right) e^{\beta_{5} t}
$$

That is,

$$
\begin{aligned}
& \left\|S_{2}\left(T, z_{0}\right)\right\|_{\mathscr{H}_{2+\sigma}}^{2} \\
& \quad=\frac{1}{2}\left(\left\|w^{\epsilon}(T)\right\|_{2 \theta+\sigma}^{2}+\left\|w_{t}(T)\right\|_{V_{\theta+\sigma}}^{2}+\left\|\xi^{T \epsilon}\right\|_{\mu, V_{2 \theta+\sigma}}^{2}\right) \\
& \quad \leqslant N_{1},
\end{aligned}
$$

where $N_{1}=N_{1}\left(T, g,\left\|z_{0}\right\|_{\mathscr{H}_{2}}, \alpha, \delta\right)$.

The proof is complete.

Let $B_{1}^{*}$ be the bounded absorbing set obtained from Theorem 14, and

$$
\mathscr{K}_{T}^{\epsilon *}:=\Pi S_{2}\left(T, B_{1}^{*}\right)
$$

Thanks to Lemma 2 , we can obtain that $\mathscr{K}_{T}^{\epsilon *}$ is relatively compact in $L_{\mu}^{2}\left(\mathbb{R}^{+} ; V_{2 \theta}\right)$. Thus, using the compact embedding $V_{2 \theta+\sigma} \hookrightarrow V_{2 \theta}$ once again, we can get the following.

Lemma 17. Let $S_{2}(t, \cdot)$ be the corresponding solution operator of (56). Under the assumption of Lemma 16, for any $T>0$, $S_{2}\left(T, B_{1}^{*}\right)$ is relatively compact in $\mathscr{H}_{2}$.

By applying the classical theory (e.g., see $[6,20]$ ), from Lemma 3, Theorem 14, and Lemmas 15 and 17, we can gain the main result.

Theorem 18 (global attractor in $\mathscr{H}_{2}$ ). Let the nonlinearity $f$ satisfy (2)-(6), $g \in L^{2}(\Omega)$, (7)-(9) hold, and $\{S(t)\}_{t \geqslant 0}$ be the solution semigroup of (17)-(18) in $\mathscr{H}_{2}$. Then $\{S(t)\}_{t \geqslant 0}$ has a global attractor $\mathscr{A}_{1}$ in $\mathscr{H}_{2}$; namely, $\mathscr{A}_{1}$ is compact and invariant in $\mathscr{H}_{2}$ and attracts every bounded subset of $\mathscr{H}_{2}$ with respect to the $\mathscr{H}_{2}$-norm.

\section{Conflicts of Interest}

The authors declare that they have no competing interests.

\section{Authors' Contributions}

The authors read and approved the final manuscript.

\section{Acknowledgments}

This work was partly supported by the NSFC (Grants 11361053, 11561064, 11401479, and 11661071) and the Young Teachers Scientific Research Ability Promotion Plan of Northwest Normal University (NWNU-LKQN-14-6).

\section{References}

[1] C. Giorgi, J. E. M. Rivera, and V. Pata, "Global attractors for a semilinear hyperbolic equation in viscoelasticity," Journal of Mathematical Analysis and Applications, vol. 260, no. 1, pp. 8399, 2001.

[2] B. D. Coleman and W. Noll, "Foundations of linear viscoelasticity," Reviews of Modern Physics, vol. 33, pp. 239-249, 1961.

[3] C. M. Dafermos, "Asymptotic stability in viscoelasticity," Archive for Rational Mechanics and Analysis, vol. 37, pp. 297-308, 1970.

[4] M. Fabrizio and A. Morro, Mathematical problems in linear viscoelasticity, vol. 12 of SIAM Studies in Applied Mathematics, Society for Industrial and Applied Mathematics (SIAM), Philadelphia, PA, 1992.

[5] J. Arrieta, A. N. Carvalho, and J. K. Hale, "A damped hyperbolic equation with critical exponent," Communications in Partial Differential Equations, vol. 17, no. 5-6, pp. 841-866, 1992.

[6] R. Temam, Infinit Dimensional Dynamical System in Mechanichs and Physics, Springer, New York, NY, USA, 2nd edition, 1997.

[7] A. C. Lazer and P. J. McKenna, "Large-amplitude periodic oscillations in suspension bridges: some new connections with nonlinear analysis," SIAM Review. A Publication of the Society for Industrial and Applied Mathematics, vol. 32, no. 4, pp. 537578, 1990. 
[8] Q. Z. Ma, "Asymptotic behavior of the solution for the floating beam," Journal of Northwest Normal University (Natural Science), vol. 41, no. 6, pp. 4-7, 2005.

[9] V. Pata and A. Zucchi, "Attractors for a damped hyperbolic equation with linear memory," Journal of Advanced Mathematics and Applications, vol. 11, no. 2, pp. 505-529, 2001.

[10] Q. Ma and C. Zhong, "Existence of strong global attractors for hyperbolic equation with linear memory," Applied Mathematics and Computation, vol. 157, no. 3, pp. 745-758, 2004.

[11] C. Sun, D. Cao, and J. Duan, "Non-autonomous wave dynamics with memory-asymptotic regularity and uniform attractor," Discrete and Continuous Dynamical Systems. Series B. A Journal Bridging Mathematics and Sciences, vol. 9, no. 3-4, pp. 743-761, 2008.

[12] M. M. Cavalcanti, L. H. Fatori, and T. F. Ma, "Attractors for wave equations with degenerate memory," Journal of Differential Equations, vol. 260, no. 1, pp. 56-83, 2016.

[13] S. F. Zhou and M. Zhao, "Random attractors for damped nonautonomous wave equations with memory and white noise," Nonlinear Analysis, vol. 120, Article ID 10484, pp. 202-226, 2015.

[14] L. Xu and Q. Ma, "Existence of random attractors for the floating beam equation with strong damping and white noise," Boundary Value Problems, 2015:126, 13 pages, 2015.

[15] S. Borini and V. Pata, "Uniform attractors for a strongly damped wave equation with linear memory," Asymptotic Analysis, vol. 20, no. 3-4, pp. 263-277, 1999.

[16] S. Gatti, A. Miranville, V. Pata, and S. Zelik, "Attractors for semilinear equations of viscoelasticity with very low dissipation," The Rocky Mountain Journal of Mathematics, vol. 38, no. 4, pp. 11171138, 2008.

[17] C. Sun and M. Yang, "Dynamics of the nonclassical diffusion equations," Asymptotic Analysis, vol. 59, no. 1-2, pp. 51-81, 2008.

[18] X. Wang, W. C. Ju, and C. K. Zhong, "Strong attractors for the non-autonomous nonclassical diffusion equations with fading memory," Chinese Annals of Mathematics, vol. 34A, no. 6, pp. 671-688, 2013.

[19] X. Wang, L. Yang, and C. Zhong, "Attractors for the nonclassical diffusion equations with fading memory," Journal of Mathematical Analysis and Applications, vol. 362, no. 2, pp. 327-337, 2010.

[20] J. W. Cholewa and T. Dlotko, Global Attractors in Abstract Parabolic Problems, vol. 278, Cambridge University Press, 2000. 


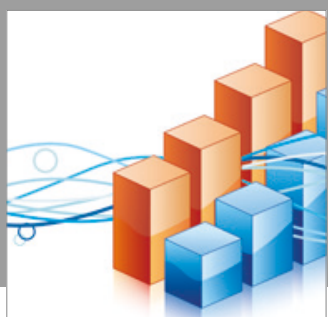

Advances in

Operations Research

vatersals

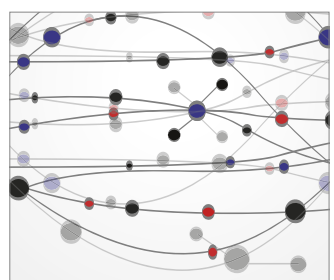

\section{The Scientific} World Journal
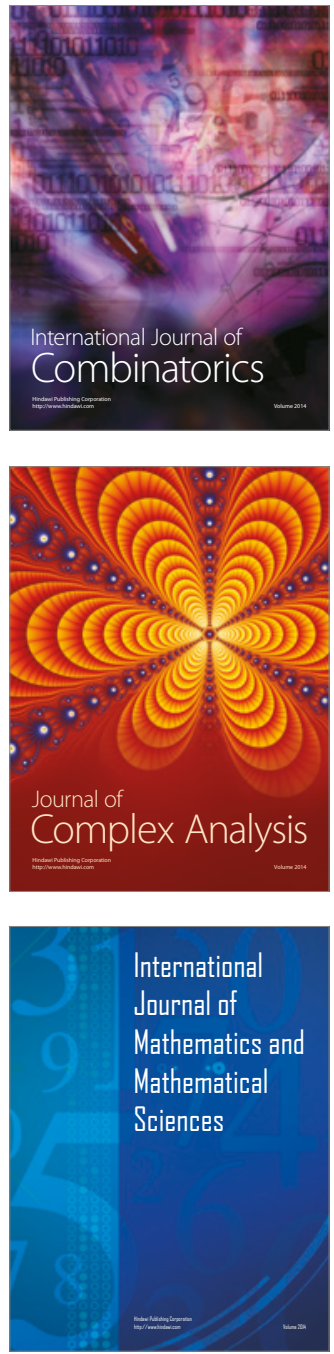
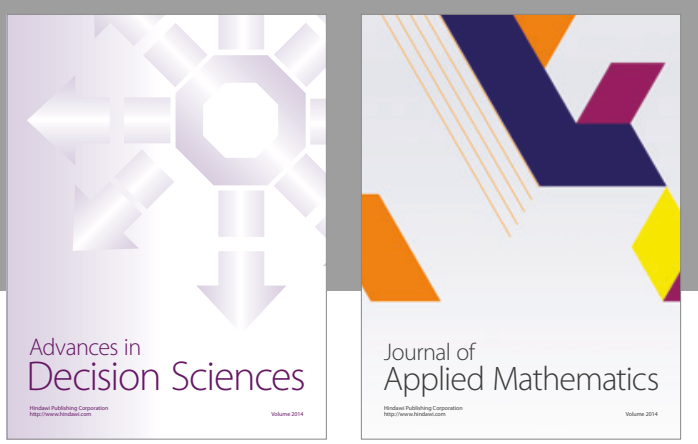

Algebra

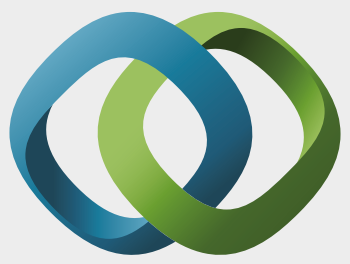

\section{Hindawi}

Submit your manuscripts at

https://www.hindawi.com
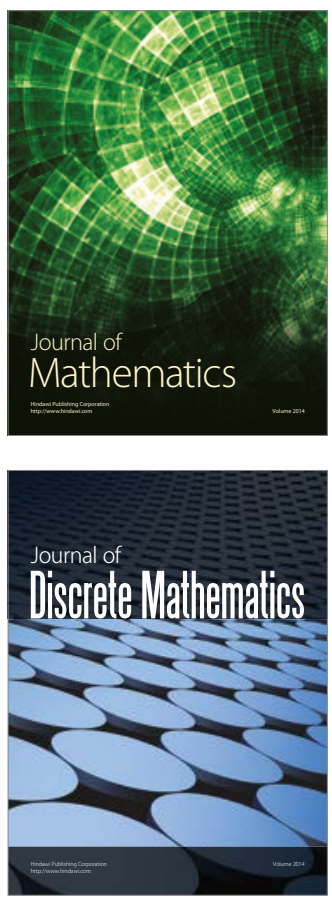

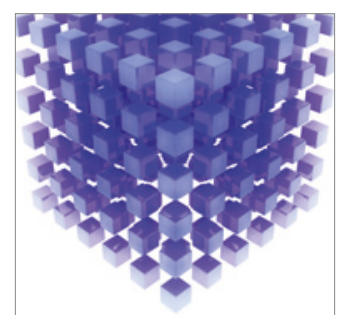

Mathematical Problems in Engineering
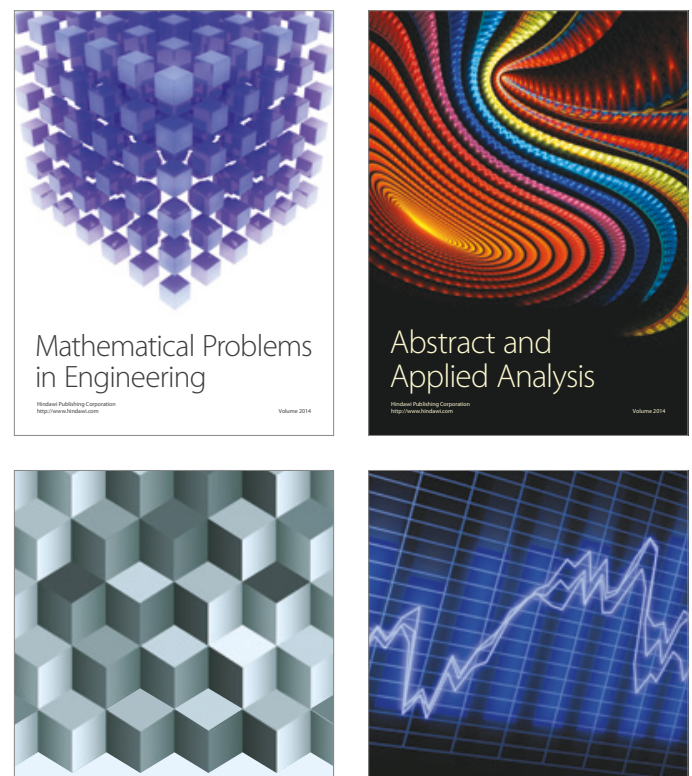

Journal of

Function Spaces

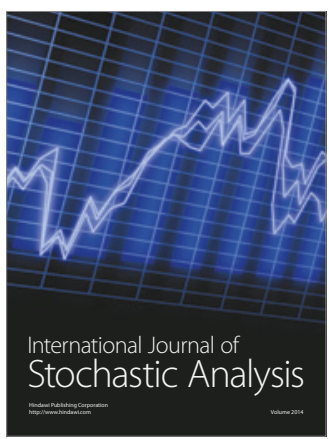

Probability and Statistics
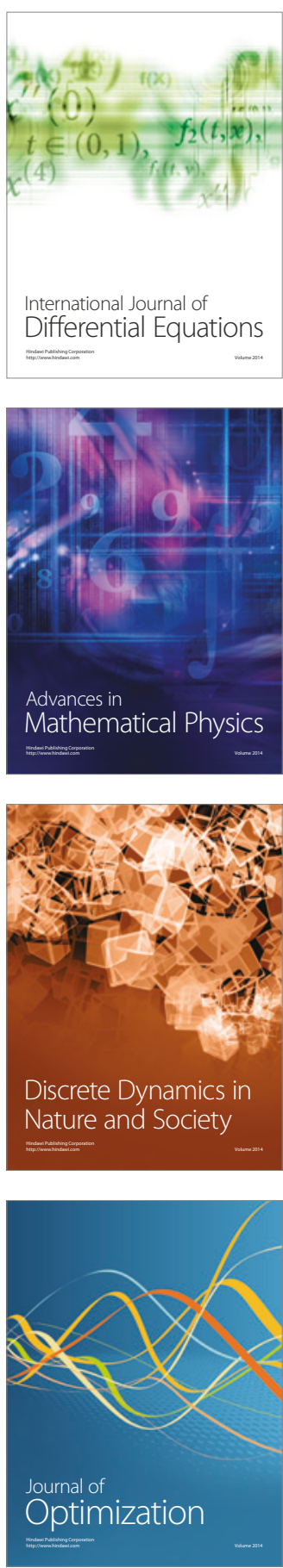